\title{
Phasic Activation of Individual Neurons in the Locus Ceruleus/Subceruleus Complex of Monkeys Reflects Rewarded Decisions to Go But Not Stop
}

\author{
Rishi M. Kalwani, ${ }^{1}{ }^{\oplus}$ Siddhartha Joshi, ${ }^{2}$ and ${ }^{\circledR}$ Joshua I. Gold $^{2}$ \\ ${ }^{1}$ Temple University School of Medicine, Philadelphia, Pennsylvania 19140, and 2Department of Neuroscience, University of Pennsylvania, Philadelphia, \\ Pennsylvania 19104
}

\begin{abstract}
Neurons in the brainstem nucleus locus ceruleus (LC) often exhibit phasic activation in the context of simple sensory-motor tasks. The functional role of this activation, which leads to the release of norepinephrine throughout the brain, is not yet understood in part because the conditions under which it occurs remain in question. Early studies focused on the relationship of LC phasic activation to salient sensory events, whereas more recent work has emphasized its timing relative to goal-directed behavioral responses, possibly representing the end of a sensory-motor decision process. To better understand the relationship between LC phasic activation and sensory, motor, and decision processing, we recorded spiking activity of neurons in the LC+ (LC and the adjacent, norepinephrine-containing subceruleus nucleus) of monkeys performing a countermanding task. The task required the monkeys to occasionally withhold planned, saccadic eye movements to a visual target. We found that many well isolated LC + units responded to both the onset of the visual cue instructing the monkey to initiate the saccade and again after saccade onset, even when it was initiated erroneously in the presence of a stop signal. Many of these neurons did not respond to saccades made outside of the task context. In contrast, neither the appearance of the stop signal nor the successful withholding of the saccade elicited an LC + response. Therefore, LC + phasic activation encodes sensory and motor events related to decisions to execute, but not withhold, movements, implying a functional role in goal-directed actions, but not necessarily more covert forms of processing.
\end{abstract}

Key words: countermanding; norepinephrine; saccade

\section{Introduction}

The locus ceruleus (LC) is the primary source of norepinephrine (NE) transmitted to the rest of the brain (Jones and Moore, 1977; Aston-Jones and Cohen, 2005). The LC-NE system contributes to numerous brain functions, including arousal, sensory-motor processing, attention, learning, and memory (Jouvet, 1969; Harley, 1987; Cahill and McGaugh, 1996; Berridge and Waterhouse, 2003; Aston-Jones and Cohen, 2005; Sara, 2009; Carter et al., 2010; Howells et al., 2012; Sara and Bouret, 2012). These contributions involve, at least in part, phasic activation of the LC to reward-predicting events, particularly when the events are surprising and thus lead to abrupt cognitive and behavioral shifts (Aston-Jones and Bloom, 1981b; Devauges and Sara, 1990; Sara and Segal, 1991; Aston-Jones et al., 1997; Bouret and Sara, 2004;

\footnotetext{
Received June 23, 2014; revised Aug. 5, 2014; accepted Aug. 8, 2014.

Author contributions: R.M.K., S.J., and J.I.G. designed research; R.M.K. and S.J. performed research; S.J. contributed unpublished reagents/analytic tools; R.M.K. and J.I.G. analyzed data; R.M.K., S.J., and J.I.G. wrote the paper.

This work was supported by the National Institutes of Health (Grants P50 MH062196, R21 MH093904, R01 EY015260, and P30 EY001583), the McKnight Endowment Fund for Neuroscience, the Burroughs-Wellcome Fund, and the Sloan Foundation. We thank Jon Cohen, Long Ding, Takahiro Doi, Siddhartha Joshi, Yin Li, and Matt Nassar for helpful comments.

The authors declare no competing financial interests.

Correspondence should be addressed to Joshua I. Gold, Department of Neuroscience, University of Pennsylvania, 116 Johnson Pavilion, Philadelphia, PA 19104-6074; E-mail: jigold@mail.med.upenn.edu.

DOI:10.1523/JNEUROSCI.2566-14.2014

Copyright $\odot 2014$ the authors $\quad 0270-6474 / 14 / 3413656-14 \$ 15.00 / 0$
}

Bouret and Sara, 2005; Corbetta et al., 2008). However, basic questions about this phasic activation remain unanswered, including its relationship to sensory and motor events and the internal decision processes that link the two (Aston-Jones and Bloom, 1981b; Rasmussen et al., 1986; Grant et al., 1988; HervéMinvielle and Sara, 1995; Usher et al., 1999; Bouret and Sara, 2004; Clayton et al., 2004; Aston-Jones and Cohen, 2005; Bouret and Richmond, 2009). The goal of the present study was to clarify these relationships by recording single-unit LC activation in monkeys performing a countermanding task that involves decisions to execute and withhold visually guided saccadic eye movements (Fig. 1; Logan et al., 1984; Hanes and Schall, 1995; Hanes et al., 1998; Hanes and Carpenter, 1999).

For the countermanding task, a visual "go" signal instructs the subject to make a visually guided saccade to a peripheral target, but an occasional visual "stop" signal instructs the subject to withhold the impending saccade. The "go" process involves a buildup of oculomotor activity toward a threshold (Carpenter and Williams, 1995; Hanes and Schall, 1996). Less is known about the "stop" process, but one well established set of models assumes that this process involves a similar rise-to-threshold mechanism that races against the go process (Logan and Cowan, 1984; Boucher et al., 2007; Verbruggen and Logan, 2009). Alternatively, the go process might simply be terminated by detection of the stop signal (Salinas and Stanford, 2013). In either case, the 


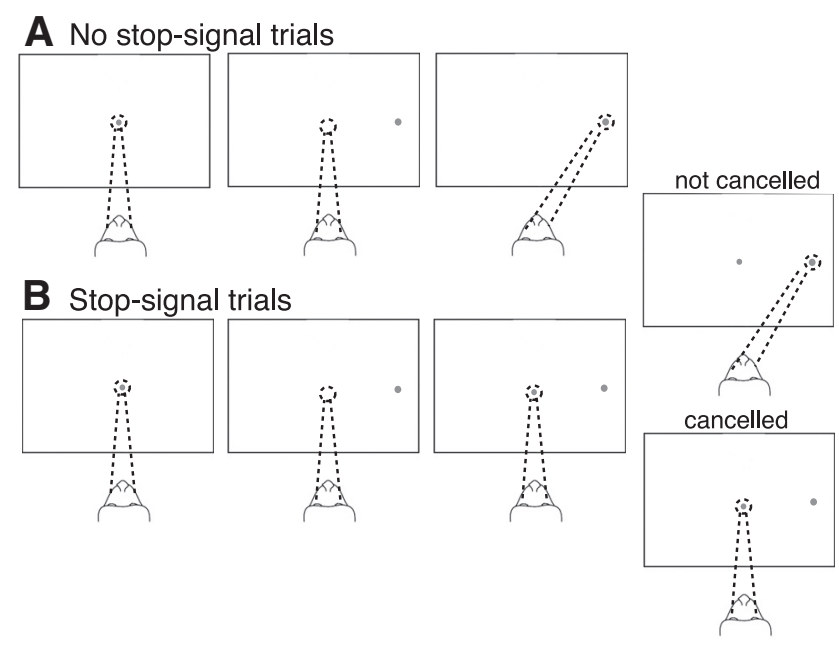

Figure 1. Countermanding task. $\boldsymbol{A}$, No-stop-signal trials. After the monkey fixated on a central fixation point for a variable time $(0.8-1.1 \mathrm{~s})$, a peripheral target appeared at 1 of 2 locations along the horizontal axis. The monkey was rewarded for making a saccade to the target. $\boldsymbol{B}$, Stop-signal trials. On 33\% of randomly selected trials, after the target appeared and after one of five stop-signal delays chosen at random, the fixation point reappeared, instructing the monkey to withhold its planned saccade. The monkey was rewarded for maintaining fixation (correct trials). If the monkey failed to withhold its saccade, no reward was given (incorrect trials).

timing of LC phasic activation can be analyzed relative to both the sensory signals and the subsequent commitments to either go or stop.

We present two novel findings that substantially clarify the conditions that elicit phasic responses in the LC and the adjacent, NE-containing subceruleus nucleus (subC; here, we refer to these nuclei together as LC+; Sharma et al., 2010; Smeets and González, 2000). First, we show that individual LC+ units can exhibit phasic activation to both sensory and motor events occurring immediately in sequence, in contrast to previous reports that typically emphasized one or the other response type, but not both (Foote et al., 1980; Aston-Jones and Bloom, 1981b; Grant et al., 1988; Hervé-Minvielle and Sara, 1995; Sara and Segal, 1991; Rajkowski et al., 2004; Aston-Jones et al., 1994; Clayton et al., 2004; but see Bouret and Richmond, 2009, which reported sensoryand motor-locked responses, albeit more separated in time). Second, we show that LC+ neurons respond phasically to rewarded decisions to go but not to stop, implying a motor-centric role that does not necessarily generalize to more covert forms of decisions.

\section{Materials and Methods}

Subjects. Two adult male rhesus monkeys (Macaca mulatta) were used for this study. All training, surgery, and experimental procedures were performed in accordance with the National Institutes of Health's Guide for the Care and Use of Laboratory Animals and were approved by the University of Pennsylvania Institutional Animal Care and Use Committee.

Behavioral task. The monkeys performed a countermanding task, which included visually guided saccade trials occasionally interrupted with a stop signal (Fig. 1). All trials began with the presentation of a central fixation point, which was a red circle subtending $2^{\circ}$ of visual angle. After the monkey fixated for a variable time $(0.8-1.1 \mathrm{~s}$, uniformly distributed), the fixation point turned off and, simultaneously, a target (a red circle subtending $3^{\circ}$ of visual angle) appeared at 1 of 2 locations either directly to the right or directly to the left at $8^{\circ}$ eccentricity (the "go signal"). Go-signal onset instructed the monkey to make a saccadic eye movement to look directly at the target. On $33 \%$ of the trials, the fixation point reappeared (the "stop signal") after a delay known as the stopsignal delay $(50,100,150,200$, or $250 \mathrm{~ms})$. Stop-signal onset instructed the monkey to withhold its saccade and maintain fixation. On stop-signal trials, the fixation point and target stimulus remained on for $400 \mathrm{~ms}$ after stop-signal onset. The monkey was rewarded with a drop of water or Kool-Aid for maintaining fixation on the fixation point throughout this time. On no-stop-signal trials, the monkey was rewarded for making a saccade to the target within $700 \mathrm{~ms}$ of fixation point offset and maintaining gaze on the target for $400 \mathrm{~ms}$.

Eye position was monitored using a video-based system (Eye-Link 2000; SR Research) sampled at $1000 \mathrm{~Hz}$. Visual stimuli were presented on an LCD monitor (Viewsonic) located $60 \mathrm{~cm}$ from the monkey's eyes. The monitor introduced systematic delays in the timing of the onset and offset of visual stimuli relative to when it received a new frame from the video card, as measured using a photodiode (OSI Optoelectronics). Because these delays remained constant across multiple measurements, we adjusted measurements of LC+ response latencies using fixed offsets equal to these delays (15 and $17 \mathrm{~ms}$ for fixation point onset and offset, respectively).

Electrophysiology. Each monkey was implanted with a head holder and recording cylinder that provided access to the $\mathrm{LC}+$ (Monkey $\mathrm{O}$ had two separate cylinders implanted at separate times, first targeting the left $\mathrm{LC}+$ and then targeting the right $\mathrm{LC}+$; Monkey $\mathrm{C}$ had a single cylinder targeting the left $\mathrm{LC}+$ ). The cylinders were placed at Horsley-Clark coordinates $0 \mathrm{~mm} \mathrm{AP}, 5 \mathrm{~mm} \mathrm{~L}$, and tilted at an angle of $9^{\circ}$ along the $\mathrm{ML}$ plane to point downward toward the midline. We used several techniques to target $\mathrm{LC}+$, including MRI to determine the trajectory of the recording chamber relative to monkey's brain (Kalwani et al., 2009); physiological properties of neurons in the brain structures encountered along recording trajectories leading to the $\mathrm{LC}+$, which included the superior colliculus, inferior colliculus (IC), and the trochlear decussation in the brainstem; characteristics of noradrenergic LC neurons reported in previous studies (Aston-Jones et al., 1994; Bouret and Sara, 2004; Bouret and Richmond, 2009), including waveform shape, sensitivity to arousing external stimuli (e.g., door knocking, key shaking), and decreased firing during observed periods of drowsiness (e.g., eyelid drooping, disengagement from behavioral tasks); and effects of intramuscular injection of the $\alpha 2$-noradrenergic agonist clonidine (Bouret and Richmond, 2009).

Neural recordings were conducted using custom-made quartz-coated platinum-tungsten microelectrodes (Thomas Recording) and a Multichannel Acquisition System (Plexon). Spike waveforms were sorted offline. Single-unit isolation quality was determined by estimating both false-positive (FP) and false-negative (FN) rates, as follows (for details, see Hill et al., 2011). FP rate was computed as the maximum of 2 values: (1) the expected rate of refractory period violations based on a refractory period of $2.6 \mathrm{~ms}$ (Segal et al., 1983) and (2) the amount of overlap between an identified signal and noise distribution each fit to a multivariate Gaussian. FN rate was computed as $(1-(1-\mathrm{FN} 1)(1-\mathrm{FN} 3)+$ FN2, where FN1 estimated false negatives based on a high threshold for spike detection, FN2 was computed from the amount of overlap between a signal and noise distribution each fit to a multivariate Gaussian, and FN3 represented "censored" events based on the time-out period of 1.4 $\mathrm{ms}$ after waveform triggering.

Behavioral data analysis. Reaction time (RT) was measured as the time between stimulus onset and saccade onset. Saccades were detected by analyzing acceleration data using an adaptive-threshold model (Behrens et al., 2010). Behavior on stop-signal trials was quantified using inhibition functions, which describe the probability of making an error as a function of stop-signal delay (Hanes and Schall, 1995). These functions were fit with a cumulative Weibull function of the following form:

$$
W(t)=\gamma+(1-\gamma-\lambda)^{*}\left(1-\exp \left(-(t / \alpha)^{\beta}\right)\right)
$$

where $t$ is the stop-signal delay, $\alpha$ is the stop-signal time at which the inhibition function reaches $80 \%$ of its growth, $\beta$ is the slope, $\gamma$ is the lower asymptote, and $\lambda$ is the upper asymptote.

Unlike for RT on "go" trials, there is no overt way to assess when the monkey has stopped. Instead, the time at which the monkey stops, known as the stop-signal reaction time (SSRT), must be inferred using the RT distribution and the inhibition function (Logan and Cowan, 1984; Hanes and Schall, 1995). The SSRT was derived by matching the 
probability of error for a given stop-signal delay from the inhibition function to the same probability on the cumulative RT function from no-stop-signal trials. The SSRT is the difference between the time associated with this probability on the cumulative RT function and the stopsignal delay.

Neural data analysis. Phasic neural activation was quantified as the spike rate measured in a 100-ms-wide window, defined separately for each unit relative to sensory or motor events. For sensory activation, the $100 \mathrm{~ms}$ response window had a start time between 50 and $100 \mathrm{~ms}$ after go-signal onset, chosen to provide the maximum spike rate from that interval. For motor activation, the $100 \mathrm{~ms}$ response window had a start time between $20 \mathrm{~ms}$ preceding and $30 \mathrm{~ms}$ after saccade onset, chosen to provide the maximum spike rate from that interval. For analyses that compared sensory and motor responses (e.g., Fig. 7), we used only trials with RT $>220$ to ensure that the sensory and motor response windows did not overlap. Because spike rates tended to be very low ( $<10$ spikes/s), responses in these $100 \mathrm{~ms}$ time windows typically consisted of either zero or one spike. We therefore used Fisher's exact test to compare responses between different windows or different task conditions for a given window.

Histology. After transcardial perfusion with $10 \%$ buffered formalin (Fisher Scientific), the brain was removed and allowed to postfix in formalin at $4^{\circ} \mathrm{C}$ for a week. It was then blocked in the coronal plane and resectioned at $60 \mu \mathrm{m}$ in $0.1 \mathrm{M}$ phosphate buffer (PB) using a Leica vibratome. Sections were collected in $0.1 \mathrm{M} \mathrm{PB}$ and rinsed twice ( $10 \mathrm{~min}, 30$ $\mathrm{min})$ in the same solution and then three times (10 min each) in $0.01 \mathrm{M}$ $\mathrm{PB}$. Alternate sections were stained using standard protocols for the Nissl procedure (on slides) and immunostaining for tyrosine hydroxylase (floating sections; primary antibody: anti-TH rabbit, AB152; Millipore). The remaining materials and methods were similar to those described previously (Sharma et al., 2010). A set of control sections was also processed without the primary antibody and found to show no labeling. All slides were dehydrated (in alcohol), defatted (in xylene), and then coverslipped using Permount (Fisher Scientific). Sections were imaged using a Leica microscope fitted with a digital camera. Montages of multiple sections for the final figures were prepared using the Gnu Image Manipulation Program (GIMP).

\section{Results}

The goal of this study was to characterize the activity of individual neurons in the LC+ of two monkeys performing a countermanding task (Fig. 1; Logan et al., 1984; Hanes and Schall, 1995; Hanes et al., 1998; Hanes and Carpenter, 1999). For this task, the monkeys were rewarded for making visually guided saccades to a peripheral target, but the saccade was withheld when a stop signal was occasionally presented after target onset (33\% of randomly interleaved trials). The targets always appeared in one of two locations along the horizontal axis, directly right or left of fixation. We varied the time between the presentation of the go signal ( simultaneous offset of the fixation point and onset of the peripheral target) and the stop signal (reappearance of the fixation point), referred to as the stop-signal delay. The task allowed us to characterize LC+ activation with respect to the visual "go" and "stop" signals, the decision to go and subsequent saccadic response, the decision to stop, and task-irrelevant movements. In the following sections, we first describe behavioral performance on the countermanding task, then detail how we targeted LC+ units, and finally describe the response properties of these units in the context of task performance.

\section{Task performance}

The performance of both monkeys on the countermanding task was consistent with results from previous studies (cf. Fig. 2A, C, with Figures 2 and 3, respectively, in Emeric et al., 2007). On no-stop-signal trials, the monkeys had median [interquartile range or IQR] RTs of 267 [233 311] ms for Monkey O and 253
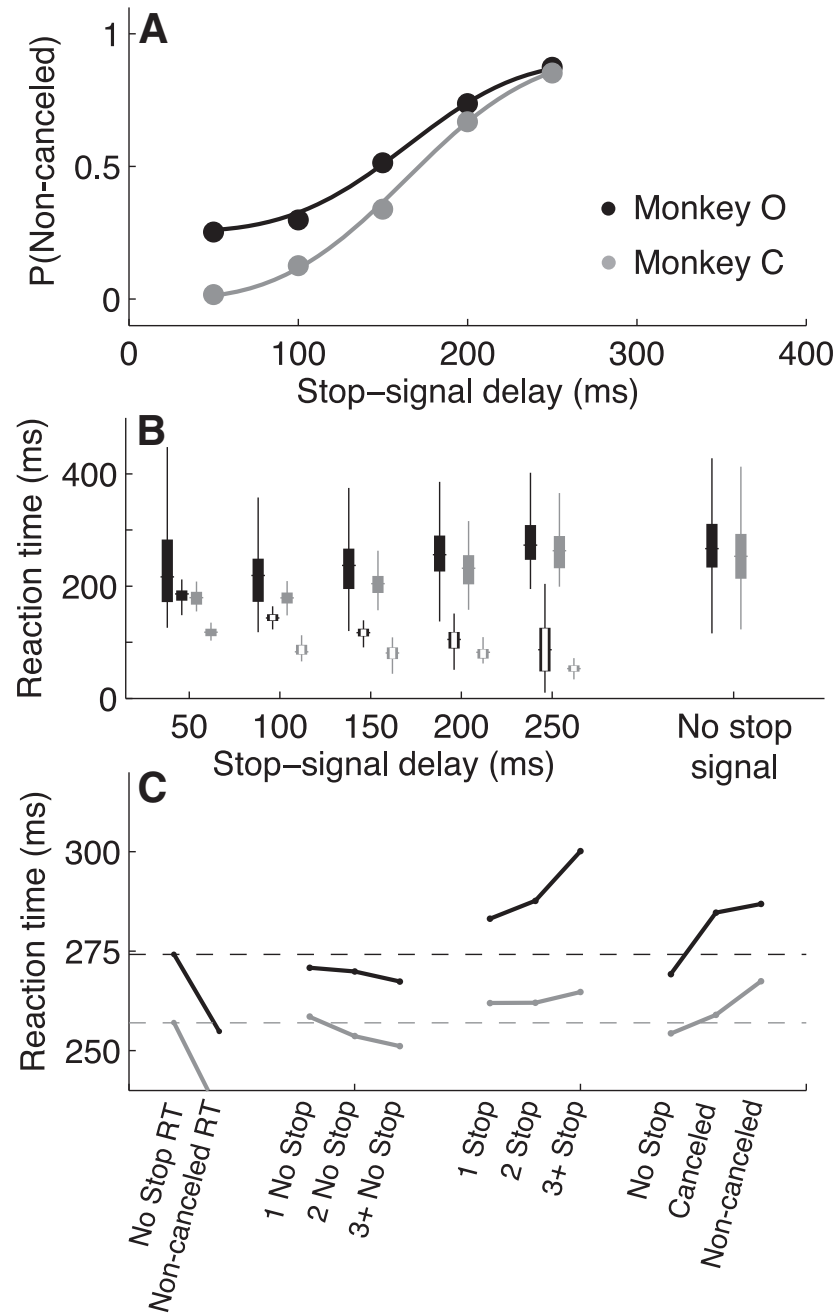

Figure 2. Behavior for Monkeys 0 (black) and C (gray). $A$, Inhibition functions describing the probability of noncancelled saccades on stop-signal trials (errors) as a function of stop-signal delay. Points and error bars (mostly obscured by points) are mean \pm SEM measured across sessions. Lines are fits of Equation 1 to data from all sessions for each monkey. $\boldsymbol{B}, \mathrm{RT}$ distributions. Box and whiskers correspond to 25th/75th percentiles and first/99th percentiles, respectively; center line indicates the median value. Filled boxes correspond to RTs on noncancelled or no-stop-signal trials; that is, trials with an overt motor response. Open boxes correspond to SSRTs (see Materials and Methods) on cancelled stop-signal trials. Black and gray symbols correspond to the two monkeys, as in $A$. C, Sequential trial effects on RT. The first column shows the mean RT on all no-stop-signal trials (also shown as the horizontal dashed line). The second column shows the mean RT on all noncancelled saccades on stop-signal trials. All other columns show the mean RT on no-stop-signal trials after the sequence of trials indicated on the abscissa.

[213 293] ms for Monkey C (Fig. 2B). On stop-signal trials, their ability to cancel a saccade decreased systematically with increasing stop-signal delay (Fig. 2A; Logan et al., 1984; Hanes and Schall, 1995; Hanes et al., 1998; Hanes and Carpenter, 1999). Their RTs on noncancelled (incorrect) stop-signal trials tended to increase with increasing stop-signal delay, reflecting more time to prepare and execute the saccadic response (Fig. $2 B$ ). SSRTs computed for each stop-signal delay using the RT distribution from no-stop-signal trials (Logan and Cowan, 1984) tended to decrease slightly as a function of stop-signal delay (Fig. 2B). RTs on no-stop-signal trials were also influenced by the recent trial history, including a tendency for shorter RTs after no-stop-signal trials and longer RTs after stop-signal trials (Fig. 2C; Rieger and Gauggel, 1999; Emeric et al., 2007; Verbruggen and Logan, 2008; Nelson et al., 2010; Pouget et al., 2011). 

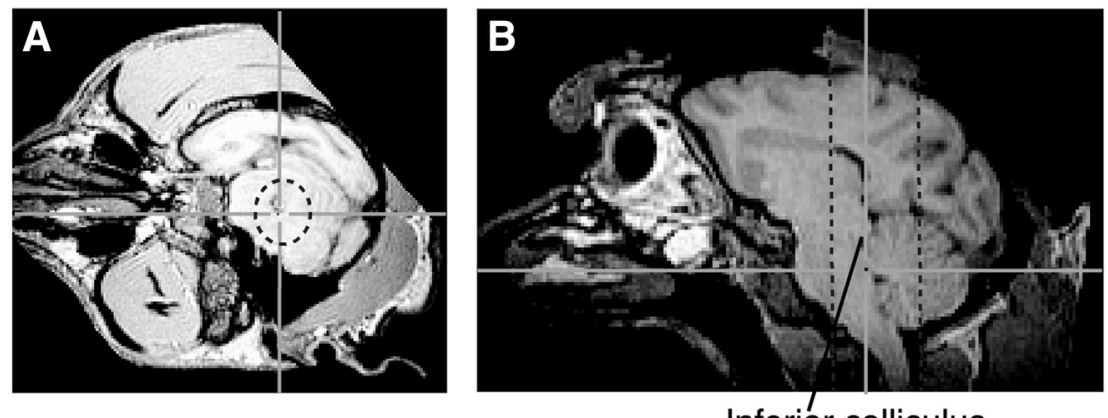

Inferior colliculus

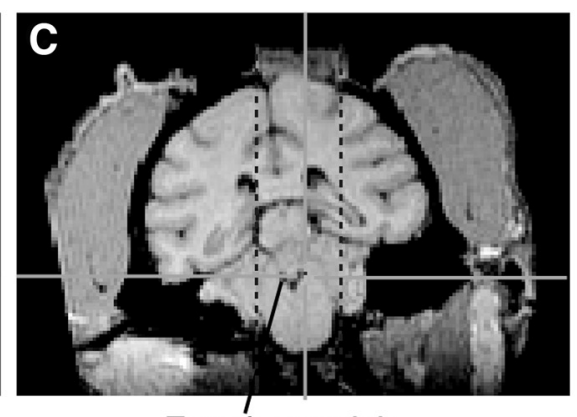

Fourth ventricle

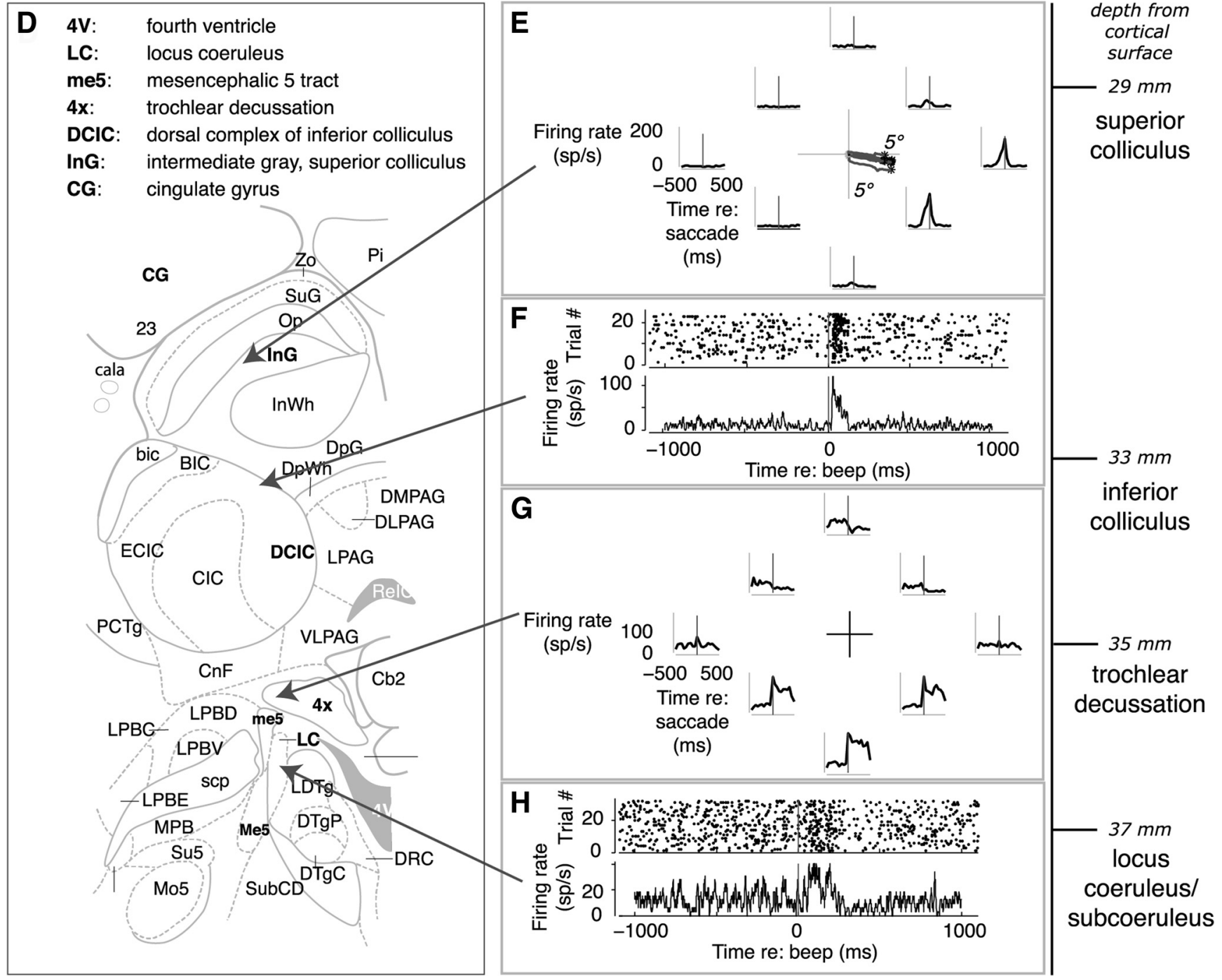

Figure 3. Targeting $\mathrm{LC}+$. $\boldsymbol{A}-\boldsymbol{C}$, Magnetic resonance images from axial $(\boldsymbol{A})$, sagittal $(\boldsymbol{B})$, and coronal $(\boldsymbol{C})$ planes showing the approximate location of the $\mathrm{LC}+($ spot added at the center of the crosshairs) in the brain of Monkey ( based on nearby landmarks including the IC (labeled in $\boldsymbol{B}$ ) and fourth ventricle (labeled in $\boldsymbol{C}$ ). Recording microelectrodes were advanced dorsal-to-ventral and slightly lateral-to-medial along the long axis of a circular recording cylinder placed on the skull. A virtual, 3D projection of the recording cylinder through the brain is shown as dashed lines (Kalwani et al., 2009). Images are shown using AFNI (Cox, 1996). D, Schematic of a coronal section of the macaque brain showing structures typically encountered in an electrode track leading to the pontine brainstem, as detailed in $\boldsymbol{E}-\boldsymbol{H}$. (Adapted from Paxinos et al., 2008, Plate 90 , Interaural 0.3 , bregma -21.60 ). $\boldsymbol{E}$, Superior colliculus. Single-unit spatial tuning on a visually guided saccade task (responses aligned to onset of saccades in the direction of the given axes relative to central fixation) and microstimulation-evoked saccades (inset at center) were to the right and slightly downward, placing the recording site at a lateral-rostral location in intermediate layer (Robinson, 1972; Sparks and Nelson, 1987). Median [IQR] interspike interval (ISI) from 42 sites recorded in our laboratory $=30$ [14 67] ms. F, IC. Raster and PSTH of a multiunit recording aligned to an unexpected auditory beep, which evoked a strong and reliable response. Median [IQR] ISI from 130 sites recorded in our laboratory $=21$ [855] ms. G, Trochlear decussation. Multiunit activity during a visually guided saccade task, plotted as in $\boldsymbol{E}$. This site had a characteristic ramp-and-hold response for saccades to locations in the lower hemifield (Henn et al., 1982). Median [IQR] ISI from 10 sites recorded in our laboratory $=11$ [7 19] ms. $\boldsymbol{H}, \mathrm{LC}+$ multiunit recording. Raster and PSTH of a multiunit recording aligned to an unexpected auditory beep, which evoked a phasic response followed by a brief suppression of tonic activity. Median [IQR] ISI from 112 sites recorded in our laboratory $=53$ [20157] ms. 


\section{Targeting LC+}

We used several techniques to target LC+ neurons for recording. Here, we present these techniques in detail to support our contentions that: (1) our neural data were from LC+ and (2) for this dataset, we were unable to distinguish LC from subC (Paxinos et al., 2008; Sharma et al., 2010). First, we used MRI to estimate LC+ location based on several local landmarks, including the IC and the fourth ventricle (Fig. $3 A-C$ ). Second, during recording sessions, we verified appropriate penetration trajectories using stereotyped patterns of responses along our electrode penetrations through the superior colliculus, IC, and brainstem structures, including the trochlear decussation (Fig. 3D-H). In the superior colliculus, we paid particular attention to trajectories of saccades evoked with electrical microstimulation, which we used to map our location within this nucleus (Robinson, 1972). Third, we identified LC+ neurons based on previously reported characteristics (AstonJones et al., 1994; Bouret and Sara, 2004; Bouret and Richmond, 2009): broad, biphasic waveforms (for all recorded units, the time from the negative to the positive peak of the average waveform had a median [IQR] value of 488 [413 575] $\mu$ s for Monkey $\mathrm{O}$ and 450 [375 500] $\mu$ s for Monkey C; by comparison, the average waveforms of 10 representative single units in the IC had widths of 390 [220 412] $\mu \mathrm{s}$, and 10 in the SC of 245 [175 315] $\mu \mathrm{s}$ ); low spontaneous firing rate (during an $800 \mathrm{~ms}$ epoch just before fixation point offset on the visually guided saccade task, there were $0-2$ spikes recorded on $78.7 \%$ of all trials at all sites in Monkey O and $92.7 \%$ in Monkey C); $<4.4 \mathrm{~mm}$ distance from IC along the dorsoventral electrode trajectories (median $[\mathrm{IQR}]=1.80[1.37$ 2.38] mm for Monkey $\mathrm{O}$ and 2.25 [1.43 3.01] mm for Monkey C); sensitivity to arousing external stimuli (e.g., door knocking, key shaking); and decreased firing during observed periods of drowsiness (e.g., eyelid drooping, disengagement from behavioral tasks). Fourth, three units were pharmacologically confirmed as consistent with LC + physiology: a $5 \mu \mathrm{g}$ intramuscular injection of the $\alpha 2$-noradrenergic agonist clonidine, which is one-quarter of the dose delivered in previous studies (Bouret and Richmond, 2009), led to a lower baseline firing rate and concurrent drowsiness (Fig. 4).

The above techniques have been used to identify units in LC but not, to our knowledge, in subC of nonhuman primates (Aston-Jones et al., 1994; Bouret and Sara, 2004; Bouret and Richmond, 2009). The subC is located just adjacent to the LC and therefore could have easily been targeted by our electrode trajectories (Paxinos et al., 2008; Sharma et al., 2010). After our recording experiments, we examined these trajectories in more detail using histological reconstructions from one of the monkeys (Monkey O). As shown in Figure 5, we believe that at least some of our trajectories are most consistent with targets in the subC, not the LC. In Monkey C, we subsequently did more precise electrophysiological mappings around putative LC+ units and found similar waveform shapes and response properties (i.e., all conforming to our LC+ identification criteria described in the previous paragraph) separated by up to $\sim 1 \mathrm{~mm}$ in brain-surface coordinates along a medial-lateral axis and up to $\sim 3 \mathrm{~mm}$ along the rostral-caudal axis, which presumably would correspond to both LC and subC (Paxinos et al., 2008). These mapping experiments also confirmed that nearby electrode tracts that did not end up in structures with LC+ properties also did not encounter the stereotypical patterns of responses through other structures (e.g., superior colliculus, IC, and brainstem structures) that we reliably found when targeting $\mathrm{LC}+$. Together, these findings suggest that we sampled both LC and subC in our recordings $(n=69$ distinct LC+ sites, consisting of 43 and four from the left and right hemisphere, respectively, of Monkey $\mathrm{O}$, and 22 from the left hemisphere of Monkey C). Because we did not identify any systematic differences in the criteria used to identify recording sites for the two monkeys, for all subsequent analyses, we present their data combined together.

We analyzed two sets of neural (spiking) data that were obtained from the same raw recording signals in individual sessions but differed in terms of single-unit isolation quality. For one set, which we refer to as "single-unit data," we used conservative spike-sorting criteria to isolate 64 single units (one pair was isolated on a single electrode; the remainder were isolated alone), thereby allowing us to characterize how single LC+ neurons encode multiple aspects of task performance. Figure $6 \mathrm{~A}$ shows estimates of false-positive and false-negative rates for these units (Hill et al., 2011). The false-positive rates had median [IQR] values of 0.01 [0.00 0.06], implying that it was unlikely that the analyses of the response properties of these units were corrupted by contributions of other units. However, in these cases, we also likely underestimated firing rates, because false-negative rates were higher $\left(0.23\right.$ [0.06 0.90], paired test for $H_{0}$ : equal medians of false-positive and false-negative rates, $p<0.01$ ).

The second dataset, which we refer to as "multiunit data," included as spikes all waveforms that crossed a threshold defined during the recording session for each of the 69 recording sites. These data thus likely included the activity from several local neurons, possibly including those not in the LC+. These multiunit signals yielded spike rates that were 1.0-52.3 times greater than the corresponding single-unit signals recorded at the same time (Fig. 6B).

\section{No task-related tonic LC+ activation}

The LC exhibits at least two distinguishable modes of activation in animals performing simple sensory-motor tasks (Aston-Jones and Bloom, 1981a; Aston-Jones and Cohen, 2005). In tonic mode, ongoing activity that is either too low or too high can reflect drowsiness or distractibility, respectively, with intermediate levels necessary for attentive engagement in goal-directed behavior (Aston-Jones et al., 1999; Usher et al., 1999). In phasic mode, short bursts of action potentials occur in relation to taskrelevant events. The phasic activation is strongest with intermediate levels of background tonic activity.

We found no evidence for task-related modulation of tonic activity. We measured LC+ tonic activity for each trial as the 


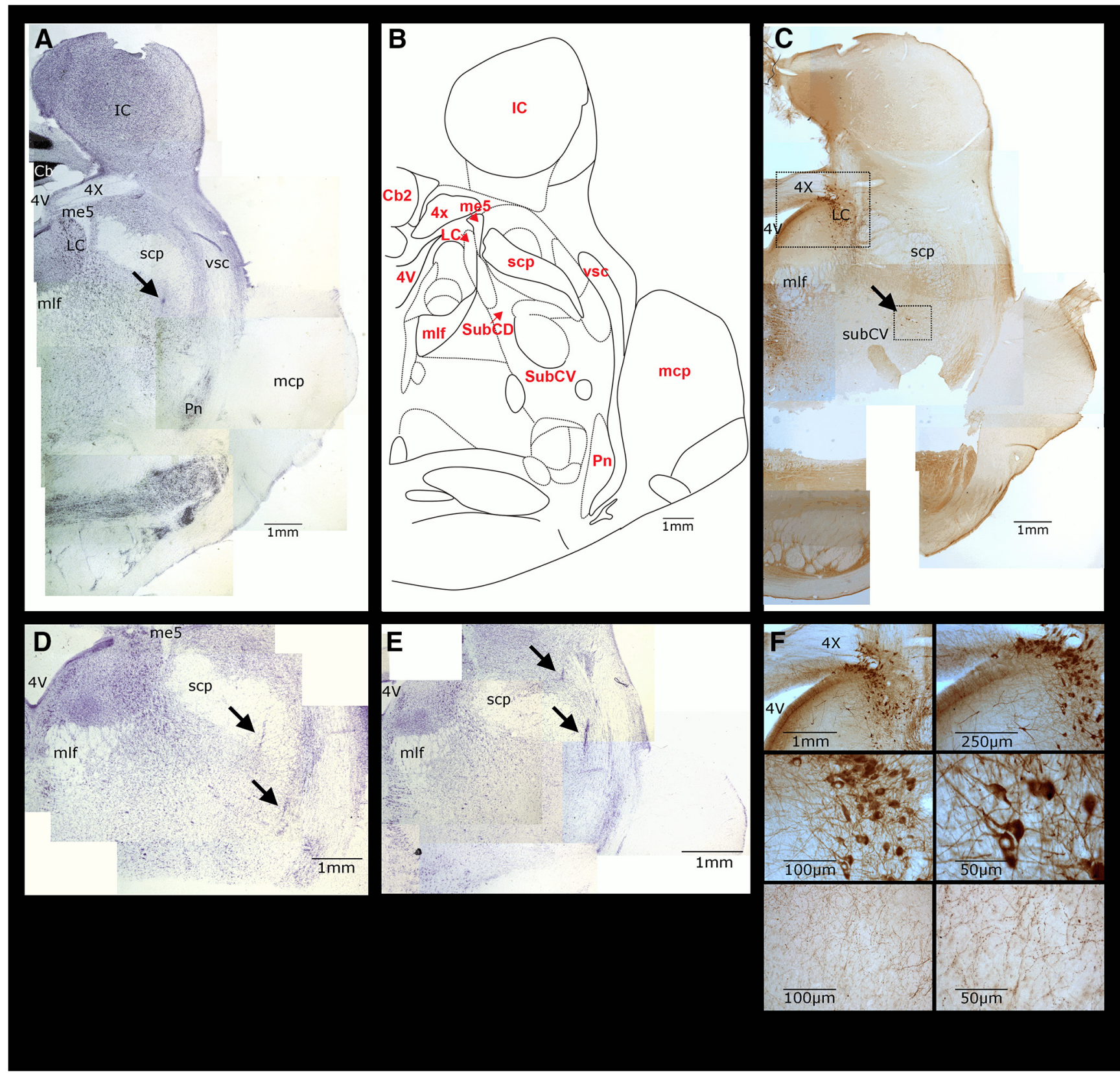

Figure 5. Histology from the right hemisphere of Monkey $0 . A$, Nissl staining used to identify brain regions at the level of the pontine brainstem. Arrow shows a small section of track and lesion visible just ventral to the superior cerebellar peduncle (scp). The track leads to subC. $\boldsymbol{B}$, Schematic showing brainstem regions that were identified in $\boldsymbol{A}$ and $\boldsymbol{C}$. (Adapted from Paxinos et al., 2008, Plate 90 , Interaural $0.3 \mathrm{~mm}$, bregma: $-21.60 \mathrm{~mm}$ ). C, Tyrosine hydroxylase (TH) immunohistochemistry allowed us to identify the LC and subCV, the only two regions with neuronal staining. This section was adjacent to the one shown in $\boldsymbol{A}$. $\boldsymbol{D}, \boldsymbol{E}$, Electrode tracks (indicated by arrows) in Nissl-stained sections adjacent to the one shown in $\boldsymbol{A}$. $\boldsymbol{F}$, Upper four panels show higher-magnification images of the region within the larger bounding box in $C$, which includes neuronal staining localized to LC. Bottom two panels show TH-immunostained axons in cortex. $4 \mathrm{~V}$ indicates fourth ventricle; $4 \mathrm{x}$, trochlear decussation; $\mathrm{Cb} / \mathrm{Cb} 2$, cerebellum; LV, lateral ventricle; $\mathrm{mcp}$, middle cerebellar peduncle; $\mathrm{me} 5$, trigeminal mesencephalic nucleus; mlf, medial longitudinal fasciculus; $\mathrm{Pn}$, pontine nuclei; $\mathrm{scp}$, superior cerebellar peduncle; subCD/subCV, dorsal/ventral parts of the subceruleus; and vsc, ventral spinocerebellar tract.

spike rate during the final $800 \mathrm{~ms}$ before fixation point offset. The mean spike rate during this epoch was the same on correct and incorrect-stop-signal trials (paired $t$ test for $H_{0}$ : equal means per session, $p=0.50$ for single-unit data, 0.16 for multiunit data) and was not related to RT on no-stop-signal trials (mean \pm SEM correlation coefficient across sessions $=-0.01 \pm 0.01, t$ test for $H_{0}$ : mean $=0, p=0.33$ for single-unit data; $-0.02 \pm 0.01, p=$ 0.11 for multiunit data) or on incorrect-stop-signal trials (correlation coefficient $=-0.01 \pm 0.02, p=0.74$ for single-unit data; $0.00 \pm 0.02, p=0.99$ for multiunit data). These results reflected a relatively constant, low-level of LC + tonic activation evident throughout all of our recording sessions (median [IQR] tonic rate across all neurons and all trials $=1.25\left[\begin{array}{ll}0 & 2.50\end{array}\right]$ spikes/s for single-unit activity and $5.00[1.2512 .00]$ spikes/s for multiunit data) when the monkeys were consistently engaged with the task. Therefore, subsequent analyses focus on LC + phasic activation.

\section{Task-related phasic LC+ activation}

Previous studies have variously emphasized either sensory or motor aspects of LC phasic activation (Berridge and Waterhouse, 2003; Clayton et al., 2004). We found that many LC+ units provided distinct, phasic responses to both sensory and motor 
events. An example single unit from Monkey $\mathrm{O}$ is shown in Figure 7. This unit had a composite false-positive rate of 0.014 and a composite false-negative rate of 0.058 . For all trial types, the "go" signal (simultaneous offset of the fixation point and onset of the peripheral target) evoked a phasic activation that peaked at a latency of $\sim 130 \mathrm{~ms}$. A second, distinct phasic activation occurred $\sim 40$ ms after the onset of the saccadic response on no-stop-signal and incorrect-stop-signal trials. However, no activation was seen on correct-stopsignal trials around the time of the SSRT, which is the time that the monkey commits to withholding the response and is inferred from the RT distribution on no-stop-signal trials and the inhibition function (Logan and Cowan, 1984; Hanes and Schall, 1995). Therefore, this unit encoded the go signal and the decision to go, but not the decision to stop.

The population of 64 recorded single LC + units had a similar combination of sensory and motor responses (Fig. 8). On average, the largest phasic activation occurred just after the onset of the visually guided saccade on both no-stop-signal and incorrectstop-signal trials. There was also a slightly smaller phasic activation that occurred $\sim 100-200 \mathrm{~ms}$ after the onset of the go signal on all trial types. There was no apparent activation after this sensory-driven activation on correct-stop-signal trials, when the monkey withheld its planned saccade. In the following sections, we analyze these sensory- and motor-related phasic activations in more detail.

\section{Single and multiunit activation to both sensory and motor events}

On no-stop-signal trials, the sensory "go" signal and the subsequent saccadic response were both associated with time-locked phasic LC+ activation (Fig. 9). Figure 9A shows average perievent time histograms (PETHs) of data from all single units, aligned to either the go signal (gray) to show sensory-related activation or the onset of the saccadic response (black) to show motor-related activation. Qualitatively, this average motor response was about twice as large as the average sensory response. Both were relatively brief, with the motor response peaking $0-100 \mathrm{~ms}$ after saccade onset and the sensory response peaking $100-200 \mathrm{~ms}$ after go-signal onset.

There was a similar combination of sensory and motor responses at most individual recording sites. Of the 64 single units, 39 had responses time locked to go-signal onset that were greater than baseline (Fisher's exact test for $H_{0}$ : equal probabilities of a spike occurring in the baseline interval and of a spike occurring in the sensory interval, $p<0.01$; one had responses lower than baseline; see Materials and Methods for details); 56 had responses time locked to saccade onset that were greater than baseline $(p<$ 0.01 ; two had responses lower than baseline); and 35 had sensoryand motor-related responses (using nonoverlapping epochs) that were both greater than baseline $(p<0.01$; Fig. $9 B)$. The mean motor response was larger than the mean sensory response from individual units, corresponding to a median [IQR] ratio of 1.63 [0.88 3.39] (Mann-Whitney test for $H_{0}$ : median ratio $=1, p<$ $0.01)$.
The sensory and motor responses were evident together in individual trials. Because overall spike rates were so low, a "response" in the given $100 \mathrm{~ms}$ epoch typically consisted of only a single spike. Figure $9 \mathrm{C}$ shows, for each single unit, the probability of a spike occurring in the motor epoch plotted as a function of the probability of a spike occurring in the motor epoch given that a spike had already occurred in the immediately preceding sensory epoch from the same trial. This analysis used only trials with RT $>220$ to ensure that the sensory and motor response epochs did not overlap. The data cluster around the main diagonal, indicating similar values for a given unit (paired $t$ test for $H_{0}$ : mean difference $=0, p=0.61$ ). Therefore, individual LC+ units can consistently provide both sensory- and motor-related phasic activation in the context of a simple visually guided saccade task.

Our conservative spike-sorting criteria for single units did not result in missed features of the neural responses. Figure 9 , $D-F$, shows the same analyses as in Figure 9, $A-C$, but using the neural recordings sorted as multiunit data. Other than substantially higher firing rates, the multiunit data showed the same trends as the single-unit data, including a combination of sensory responses and slightly larger motor responses from individual trials and recording sites. The similarity between single and multiunit responses were apparent in all subsequent analyses, so for the sake of brevity, only single-unit data are shown in subsequent figures.

Only a small number of units exhibited spatial selectivity for either sensory- or motor-related responses. Spatial tuning was measured coarsely by comparing responses on correct no-stopsignal trials when the target was either to the left (ipsilateral to the recording site in both monkeys) or right (contralateral) of fixation. For sensory responses, spatial selectivity was significant for six units showing a contralateral bias and for one unit showing an ipsilateral bias (Fisher's exact test comparing the presence/absence of a spike in the sensory epoch for the contralateral versus ipsilateral target location, $p<0.01)$. The population as a whole did not show a bias (median [IQR] difference for contralateral versus ipsilateral targets $=0.13[-0.331 .01]$ spikes $/ \mathrm{s}$ across all single-unit data; paired Mann-Whitney test for $H_{0}$ : median difference $=0, p=0.44$; Fig. 10A). For motor responses, spatial selectivity was significant for seven units showing a contralateral 


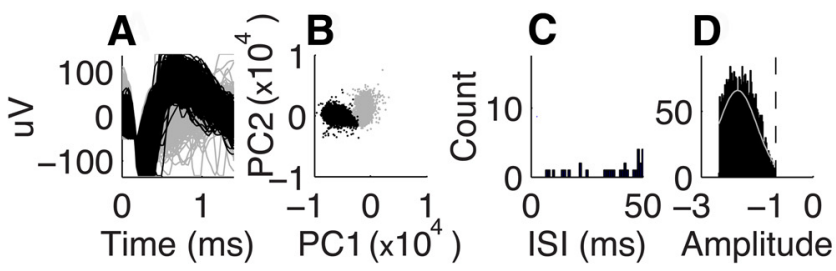

E No stop-signal trials
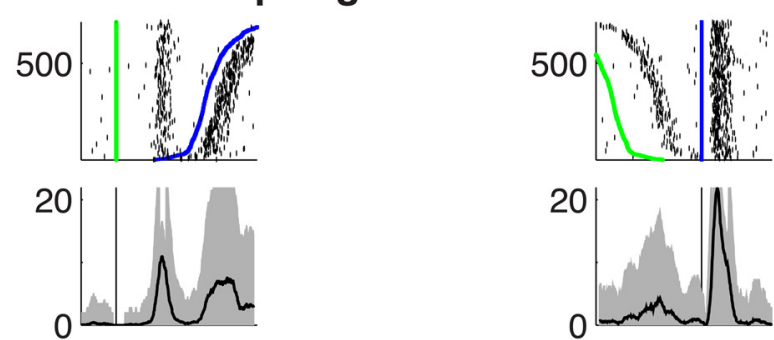

\section{F Correct stop-signal trials}
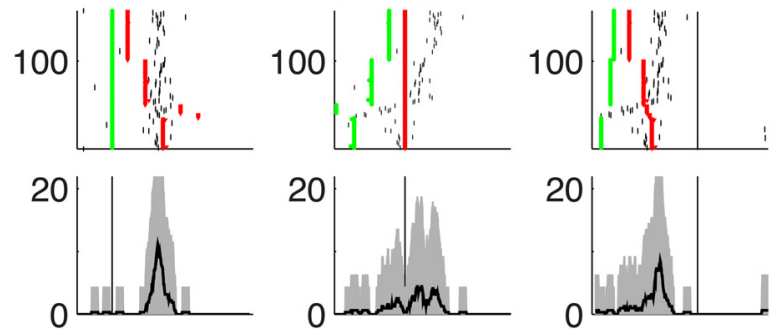

\section{G Incorrect stop-signal trials}

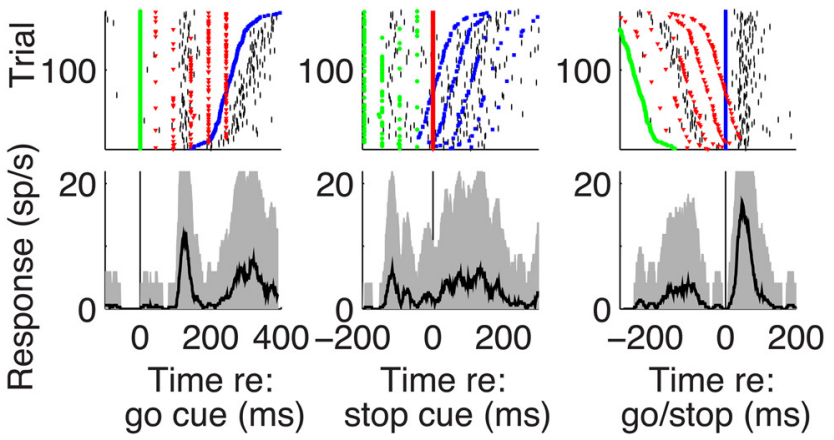

Figure 7. Single-unit example. $\boldsymbol{A}-\boldsymbol{D}$, Isolation quality. $\boldsymbol{A}$, Threshold-triggered waveforms recorded from a single electrode that were (black) and were not (gray) classified as the given unit. $\boldsymbol{B}$, Scatterplot of the first two principal components of each waveform shown in $\boldsymbol{A}$. $\boldsymbol{C}$, Histogram of interspike intervals. Intervals smaller than the estimated LC refractory period of 2.6 ms (vertical dashed line, from Segal et al., 1983) are assumed to represent false-positive categorizations. $\boldsymbol{D}$, Histogram of the amplitude of the spike trough, normalized relative to the threshold for detecting waveforms. The missing right tail of the Gaussian fit (gray curve) is assumed to represent false-negative categorizations. $\boldsymbol{E}-\boldsymbol{G}$, Raster (top panel of each vertical pair) and PETHs (computed as the mean and SD firing rate in 20-ms-wide bins, stepped in $1 \mathrm{~ms}$ increments, across all trials; bottom) of activity from no-stop-signal $(\boldsymbol{E})$, correct-stop-signal $(\boldsymbol{F})$, and incorrect-stop-signal $(\boldsymbol{G})$ trials, aligned to go-cue onset (left) and decision commitment (right, go-cue onset plus RT for no-stop-signal and incorrect-stop-signal trials, go-cue onset plus SSRT for correct-stop-signal trials). In $\boldsymbol{E}$ and $\boldsymbol{G}$, rasters are sorted by RT. In $\boldsymbol{F}$, rasters are sorted by SSRT. Green markers indicate go-cue onset, blue markers indicate saccade onset, and red markers indicate stop-cue onset.

bias and for one unit showing an ipsliateral bias $(p<0.01)$. The population as a whole showed a slight contralateral bias (median [IRQ] difference $=0.35[-0.271 .13]$ spikes $/ \mathrm{s}, p=0.02$ ). There was no systematic relationship between the direction and magnitude of spatial tuning for sensory versus motor responses (Fig. $10 C$; Pearson's correlation coefficient for contralateral-ipsilat- eral sensory and motor responses across units $=-0.11, p=0.37$ for $\mathrm{H}_{0}$ : correlation coefficient $=0$ ).

We were not able to identify a reliable trial-by-trial relationship between the probability of a sensory- or motorrelated response and RT within individual sessions: of the 64 single units, the median RT was not different (Mann-Whitney test, $p>0.01)$ on no-stop-signal trials without and with a spike in the sensory epoch for 61 units (for the remaining three units, two had slightly shorter RTs and one had slightly longer RTs on trials without an LC+ sensory spike) and in the motor epoch for 61 units (for the remaining three units, one had slightly shorter RTs and two had slightly longer RTs on trials without an LC+ sensory spike).

\section{Task-relevant and task-irrelevant motor responses}

Previous studies have shown that motor-related phasic responses in LC+ are found in association with task-relevant movements, but not in association with the same movements when they are made outside of the task context (e.g., in the intertrial interval; Yamamoto and Ozawa, 1989; Clayton et al., 2004). Our results were only partially consistent with these findings, with some individual LC+ neurons showing only task-relevant motor activation and others showing both task-relevant and task-irrelevant motor activation.

Figure $11 A$ shows PETHs for all of the single-unit data under two conditions. The first condition, identical to that shown in Figure $9 A$, corresponded to responses time locked to onset of the visually guided saccade on no-stop-signal trials. The second condition corresponded to responses time locked to the subsequent saccade (i.e., release from fixation on the visual target after reward delivery; data were similar when considering other saccades made in the intertrial interval). In both cases, there was a clear peak in the PETH $\sim 50 \mathrm{~ms}$ after saccade onset. However, the magnitude of the peak response was substantially larger for the first versus the second saccade.

The responses to task-relevant versus task-irrelevant saccades for individual, well-isolated LC + units are summarized in Figure $11 B$. Of the 64 units, 58 had task-relevant motor responses that were greater than baseline (Fisher's exact test, $p<0.01$ ) and 23 had task-irrelevant motor responses that were greater than baseline ( $p<0.01 ; 22$ of these had task-relevant responses). Of the 22 units with both task-relevant and task-irrelevant motor responses, eight had greater task-relevant responses, two had greater task-irrelevant responses, and the remaining 12 units had responses that were indistinguishable under the two conditions (Fisher's exact test, $p>0.01$ ). Both kinds of responses were found on individual trials, with the probability of a spike around the time of the task-irrelevant saccade unaffected by the presence of a spike around the time of the task-relevant saccade (Fig. 11C). Therefore, across the population, task-relevant motor responses were much more prevalent than task-irrelevant motor responses, although task-irrelevant motor responses were found reliably in a subset of well-isolated LC+ units.

There was no apparent systematic relationship between the relative strength of task-relevant and task-irrelevant motor responses and the relative strength of task-relevant sensory and motor responses across individual units. We defined a "motor-relevance index" as the difference between the taskrelevant and task-irrelevant motor response for a given unit divided by their sum. The value of this index varied from -1 , indicating strongly task-irrelevant responses, to 1, indicating strongly task-relevant responses. After the results shown in 


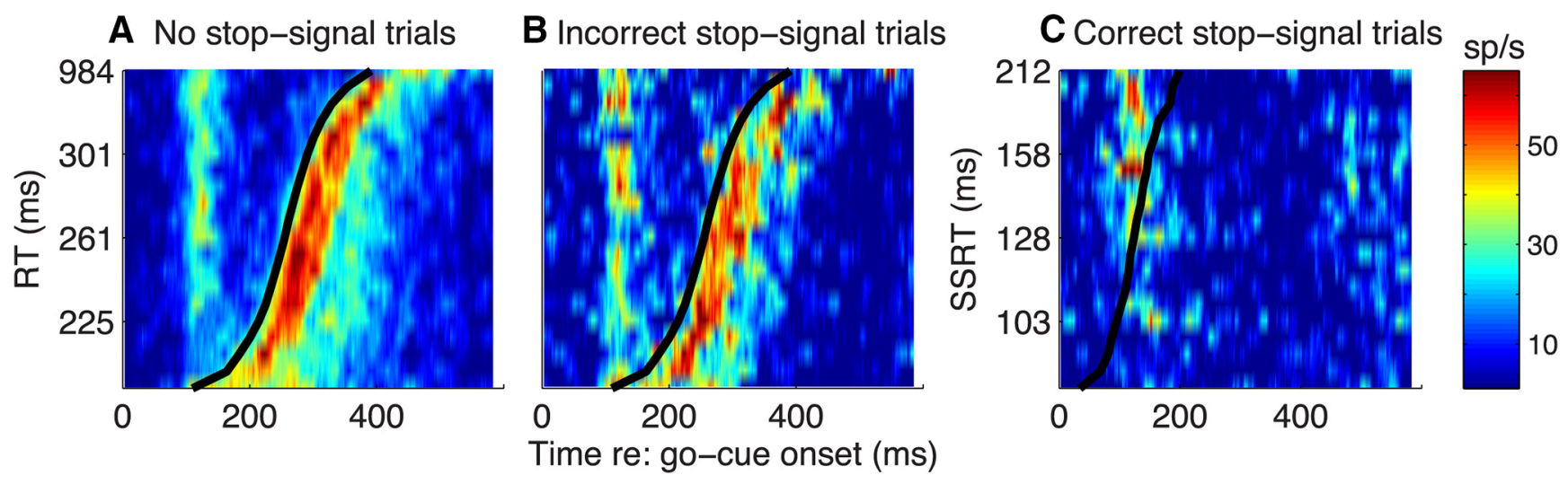

Figure 8. Pseudocolor plot of the mean response (see color scale to the right) from the full population of recorded LC+ units using trials sorted and binned by RT (black curve in $\boldsymbol{A}$ and $\boldsymbol{B}$ ) or SSRT (black curve in C) plotted as a function of time from go-cue onset. A, №-stop-signal trials. B, Incorrect-stop-signal trials. C, Correct-stop-signal trials.

A

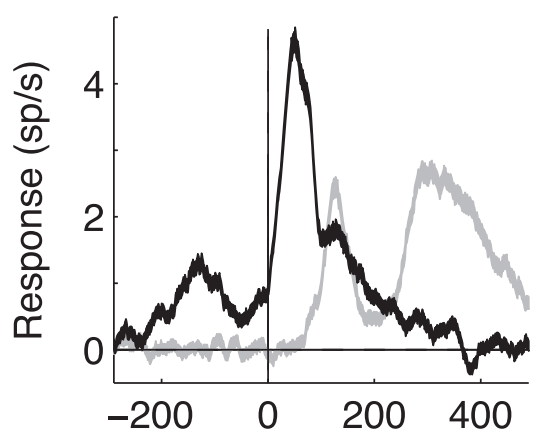

D

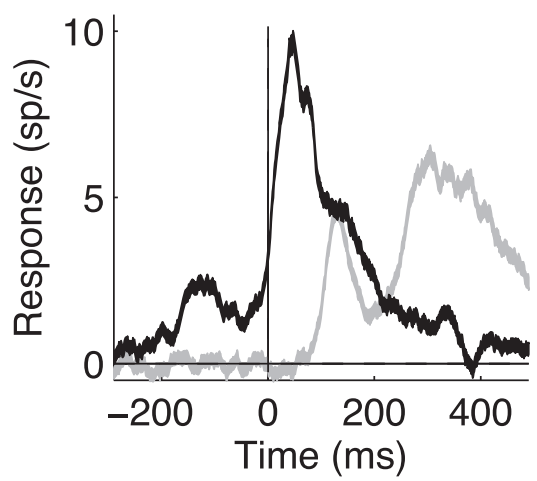

B
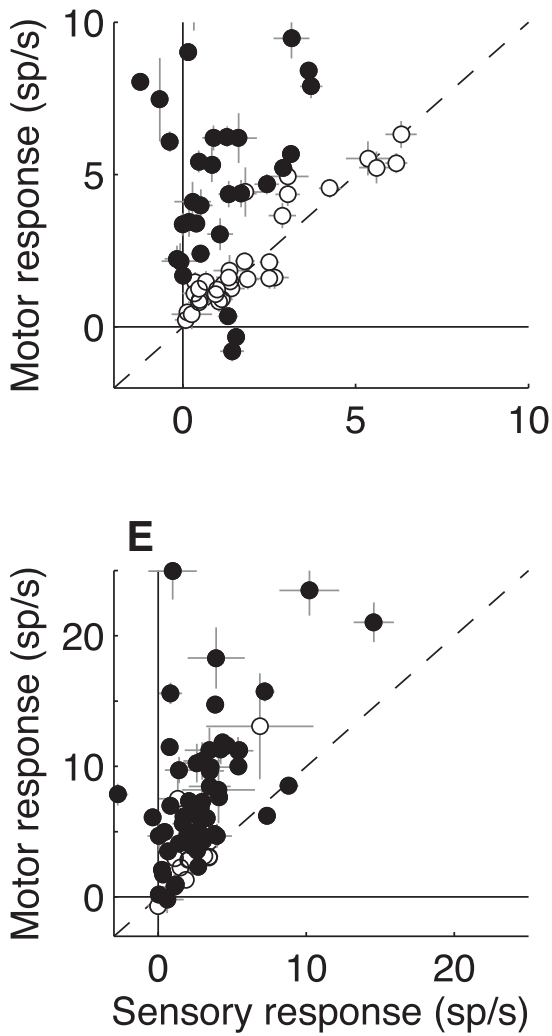
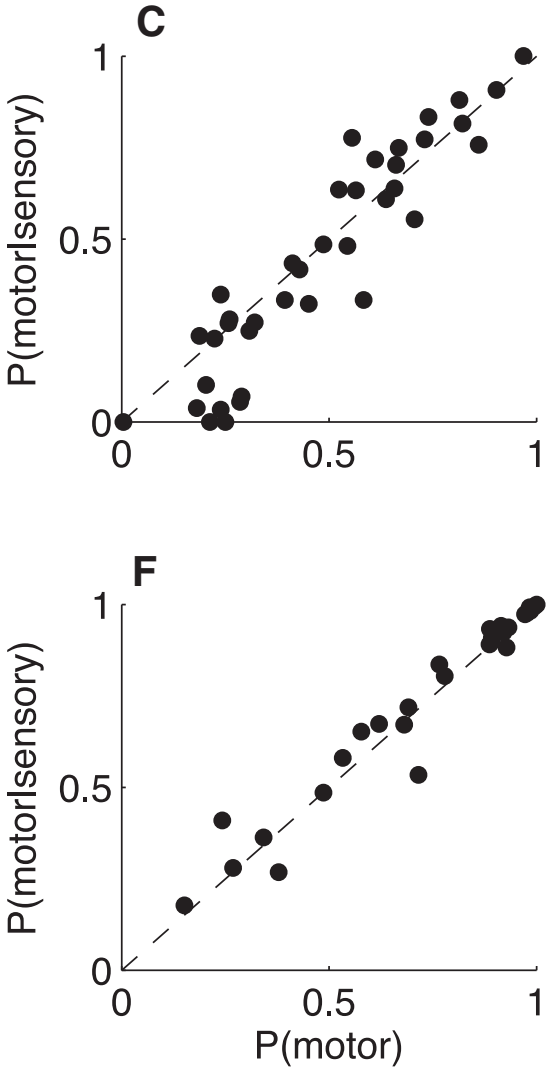

Figure 9. Summary of sensory- and motor-related phasic activations on no-stop-signal trials. $A$, PETHs of LC + activation from all single units (ribbons represent mean \pm SEM of the mean PETHs from individual units) aligned to go-cue onset (gray) or saccade onset (black). $\boldsymbol{B}$, Magnitude of the motor- versus sensory-aligned activation measured for individual units. Points and error bars are mean \pm SEM. Solid points indicate that the responses from the two epochs were significantly different from each other (Fisher's exact test, $p<0.01$ ). $C$, The probability of getting a spike in the motor epoch given that a spike occurred on the same trial in the sensory epoch plotted as a function of the overall probability of getting a spike in the motor epoch. Points represent data from individual units. $\boldsymbol{D}-\boldsymbol{F}$ are the same as $\boldsymbol{A}-\boldsymbol{C}$, except using multiunit data.

Figure $11 B$, the value of this index tended to be above zero, indicating stronger task-relevant responses (median [IQR] value $=0.21\left[\begin{array}{lll}0.06 & 0.52\end{array}\right]$ ). We also defined a "sensory-motor index" as the difference between the sensory response and the task-relevant motor response for a given unit divided by their sum. The value of this index varied from -1 , indicating strongly sensory-driven responses, to 1 , indicating strongly motor-driven responses. After the results shown in Figure $9 B$, the value of this index tended to be above zero, indicating stronger motor-related responses $($ median $[\mathrm{IQR}]$ value $=0.18$
[0.04 0.40]). The values of these indices were not correlated across units $\left(H_{0}\right.$ : Spearman's correlation coefficient $=0, p=$ 0.11 ). Instead, units strongly selective for task relevancy exhibited a range of selectivities for sensory/motor events and units strongly selective for sensory/motor events exhibited a range of selectivities for task relevancy.

We were not able to measure any topographic organization of either index. The LC+ is too small and deep in the brain to allow us to estimate the topography of our recording sites using our recording methods. 

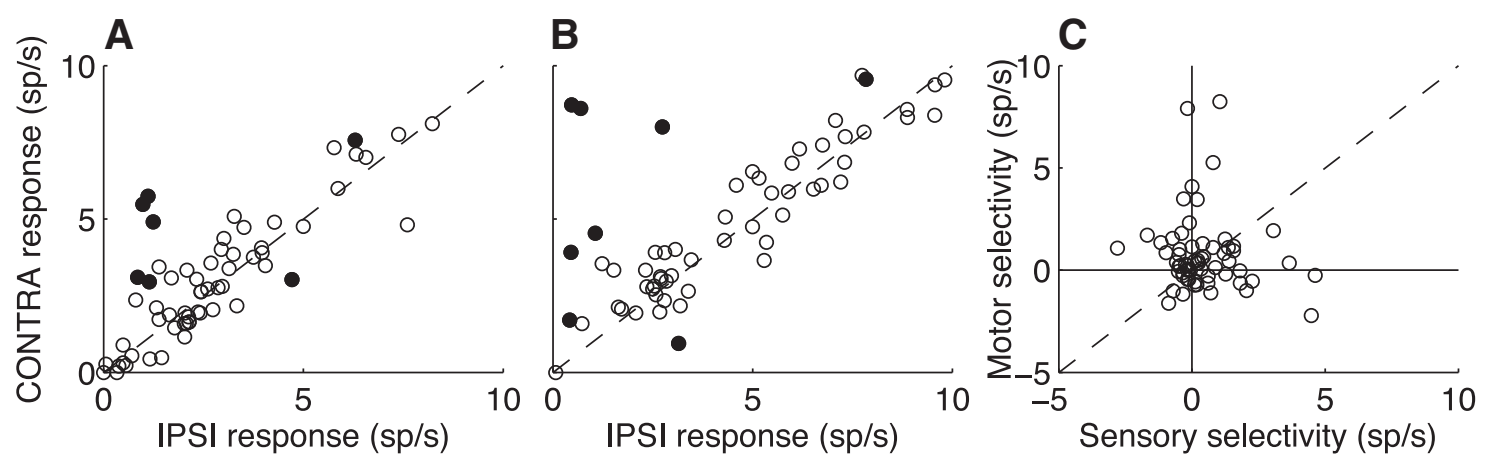

Figure 10. Weak spatial selectivity of $L C+$ phasic activation. $A$, Sensory-related spatial selectivity. Scatterplot of the mean response of individual $L C+$ units to go-cue onset when the visual target was presented to the left (ipsliateral to the recording sites) or right (contralateral) of fixation. Solid points indicate that the responses were significantly different for the two target locations (Fisher's exact test, $p<0.01)$. B, Motor-related selectivity. Same conventions as $\boldsymbol{A}$, but using responses aligned to saccade onset. $\boldsymbol{C}$, Sensory- versus motor-related selectivity. The difference in responses for contralateral versus ipsilateral targets were computed separately for sensory and motor responses of each unit.

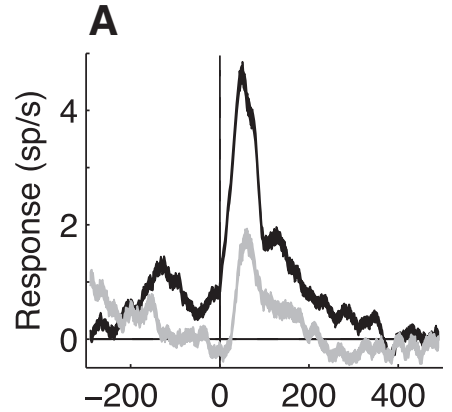

Time re: saccade onset $(\mathrm{ms})$

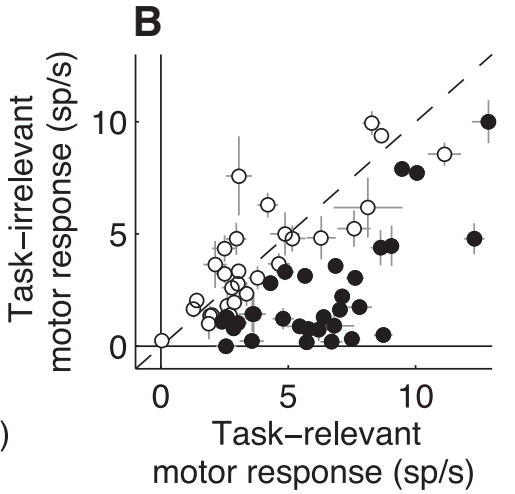

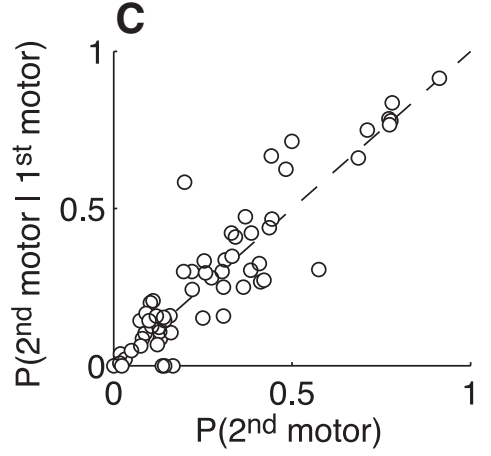

$\mathrm{P}\left(2^{\text {nd }}\right.$ motor $)$

Figure 11. Task-relevant versus task-irrelevant motor activation. $A, P E T H s$ of $L C+$ activation from all units (ribbons represent mean \pm SEM of the mean PETHs from individual units) aligned to saccade onset for task-relevant (black) versus task-irrelevant (gray) saccades. $\boldsymbol{B}$, Magnitude of the task-irrelevant versus task-relevant activity from individual units. Points and error bars are mean \pm SEM. Solid points indicate that the responses for the two conditions were significantly different from each other (Fisher's exact test, $p<0.01$ ). $C$, The probability of getting a spike in the task-irrelevant saccade epoch given that a spike occurred in the immediately preceding task-relevant saccade epoch plotted as a function of the overall probability of getting a spike in the task-irrelevant saccade epoch. Points represent data from individual units.

\section{$\mathrm{LC}+$ phasic responses on "go" decisions but not "stop" decisions}

A previous study reported phasic LC activation around the time of a rewarded motor response to a visual target, but no activation around the time of a correct but unrewarded decision to withhold the motor response to a nontarget visual stimulus (Rajkowski et al., 2004). In principle, that finding could reflect LC selectivity for either a visually guided decision to execute versus withhold a movement or the different reward contingencies. By rewarding both correctly executed and correctly withheld movements, the countermanding task allowed us to disentangle these possibilities and test directly how LC+ phasic activation relates to visually guided, rewarded decisions to go or stop.

We found clear differences between $\mathrm{LC}+$ phasic activation on trials with executed versus withheld movements. Summary PETHs from all single units for no-stop-signal trials, incorrectstop-signal trials, and correct-stop-signal trials are shown in Figure 12A. The data from no-stop-signal trials (which were rewarded) and incorrect-stop-signal trials (which were not rewarded) both show strong phasic activation peaking just after the onset of the saccadic response. Within the first $100 \mathrm{~ms}$ after saccade onset, there was no difference in response magnitude for these two trial types in 61 of the 64 recorded single units (Fisher's exact test, $p>0.01$; Fig. 12B). In the subsequent $100 \mathrm{~ms}$ interval (100-200 ms after saccade onset), there were slightly fewer (59) units with equivalent responses for no-stop-signal and incorrect- stop-signal trials (Fig. 12C), reflected in a slightly truncated response for incorrect-stop-signal trials evident in the population PETH (Fig. 12A).

In contrast, there was no apparent response on correct-stopsignal trials around the time of commitment to the stop process (i.e., at the SSRT; Fig. $12 A, D, E$ ). The majority of units had responses that did not differ from baseline (Fisher's exact test, $p<$ $0.01)$ either during the first $100 \mathrm{~ms}$ (53/64 single units) or the second $200 \mathrm{~ms}(59 / 64)$ after stop commitment. In both epochs, this lack of responsiveness differed substantially from the gorelated responses on no-stop-signal trials (Fig. 12D,E).

Moreover, the visual presentation of the stop signal did not cause a reliable $\mathrm{LC}+$ phasic activation. On incorrect-stop-signal trials, any activation in response to stop-signal onset would likely occur around the time of saccade onset, making it difficult to distinguish sensory- and motor-related activations. Nevertheless, the fact that most LC+ units had slightly weaker activation within the first $100 \mathrm{~ms}$ of saccade onset for incorrect-stop-signal trials (when the stop signal was present) versus no-stop-signal trials (when the stop signal was absent) implies a lack of responsiveness to the presentation of the stop signal (Fig. 12B).

More direct evidence comes from correct-stop-signal trials, which did not have motor-related activation after stop-signal onset. Figure 13 shows PETHs of spiking data from all recorded single units on correct-stop-signal trials. When the data are aligned to go-signal onset (gray PETHs), there is a clear activation 

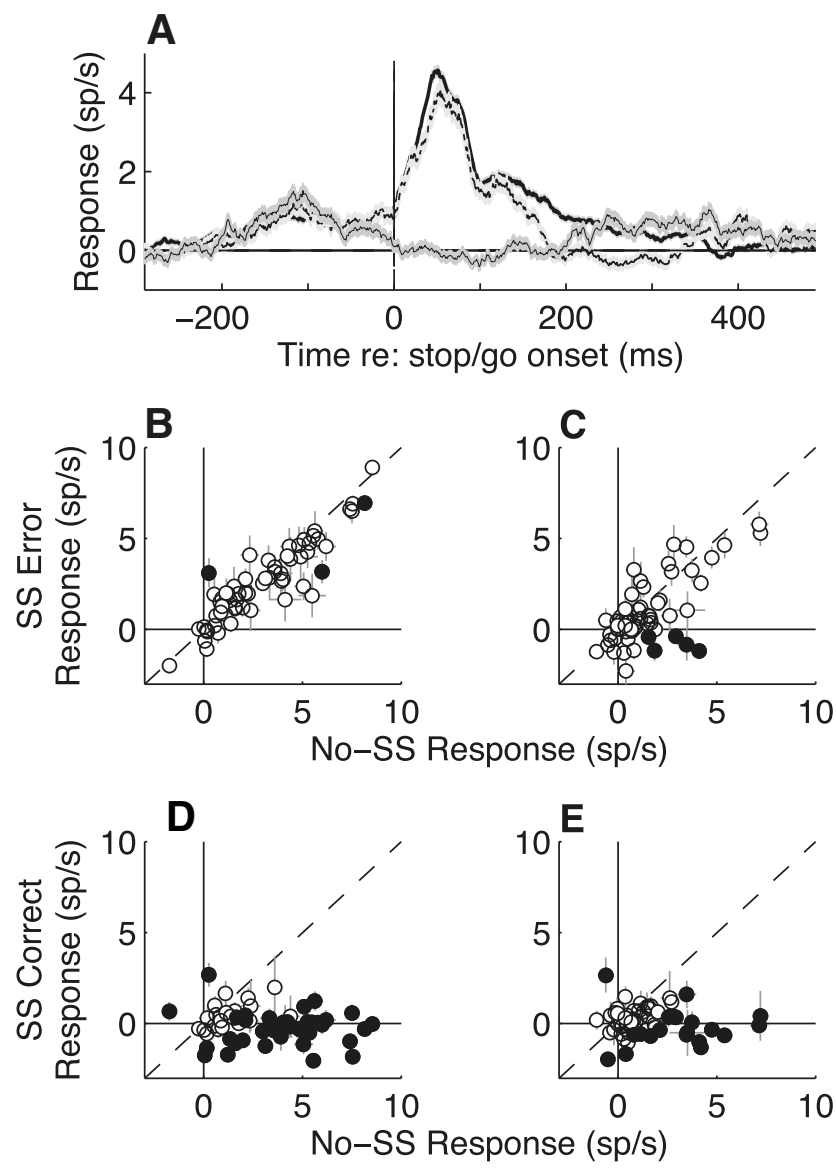

Figure 12. $\mathrm{LC}+$ activation with respect to decisions to go or stop. $A$, PETHs of $\mathrm{LC}+$ activation from all units (ribbons represent mean \pm SEM of the mean PETHs from individual units) for no-stop-signal trials (thick, solid line) and incorrect-stop-signal trials (dashed line) aligned to saccade onset and correct-stop-signal trials (thin, solid line) aligned to the inferred time of the commitment to stop (see Materials and Methods). B, C, Response magnitude from individual units (points and error bars are mean \pm SEM) on incorrect-stop-signal versus no-stop-signal trials, measured in the first $(\boldsymbol{B})$ or second $(\boldsymbol{C}) 100 \mathrm{~ms}$ epoch after go/stop onset. Solid symbols indicate that the responses were significantly different for the two conditions (Fisher's exact test, $p<0.01)$. $\boldsymbol{D}, \boldsymbol{E}$, Comparison of responses from correct-stop-signal versus no-stop-signal trials, using the same conventions as in $\boldsymbol{B}$ and $\boldsymbol{C}$.

$\sim 130$ ms later (peak values of baseline-subtracted spike rates measured between 118 and $143 \mathrm{~ms}$ after go-signal onset $=2.3$, $2.3,1.6,1.5$, and 2.7 spikes/s for the 5 SSDs shown in Fig. 13A-E, respectively). In contrast, when the data are aligned to stop-signal onset (black PETHs), there is no clear activation at the same response latency $(0.0,0.3,0.0,-0.3$, and 0.1 spikes/s in Fig. $13 A-E$, respectively; responses $90-140 \mathrm{~ms}$ after stop-signal onset were significantly smaller than responses in the same window after go-signal onset for all values of SSD, Fisher's exact test, $p<$ $0.05)$.

\section{Discussion}

We measured the responses of individual LC+ neurons in monkeys performing a simple visually guided saccade task with infrequent, randomly interleaved stop-signal trials that required the monkeys to withhold the planned saccade. We present two novel findings. The first is that many LC + neurons exhibited phasic activation to both sensory and motor events in the context of the task, with an overall dominance of the motor-related activation. The second novel finding is that $\mathrm{LC}+$ phasic activation corresponded to the reward-driven decision to act, but not the reward-
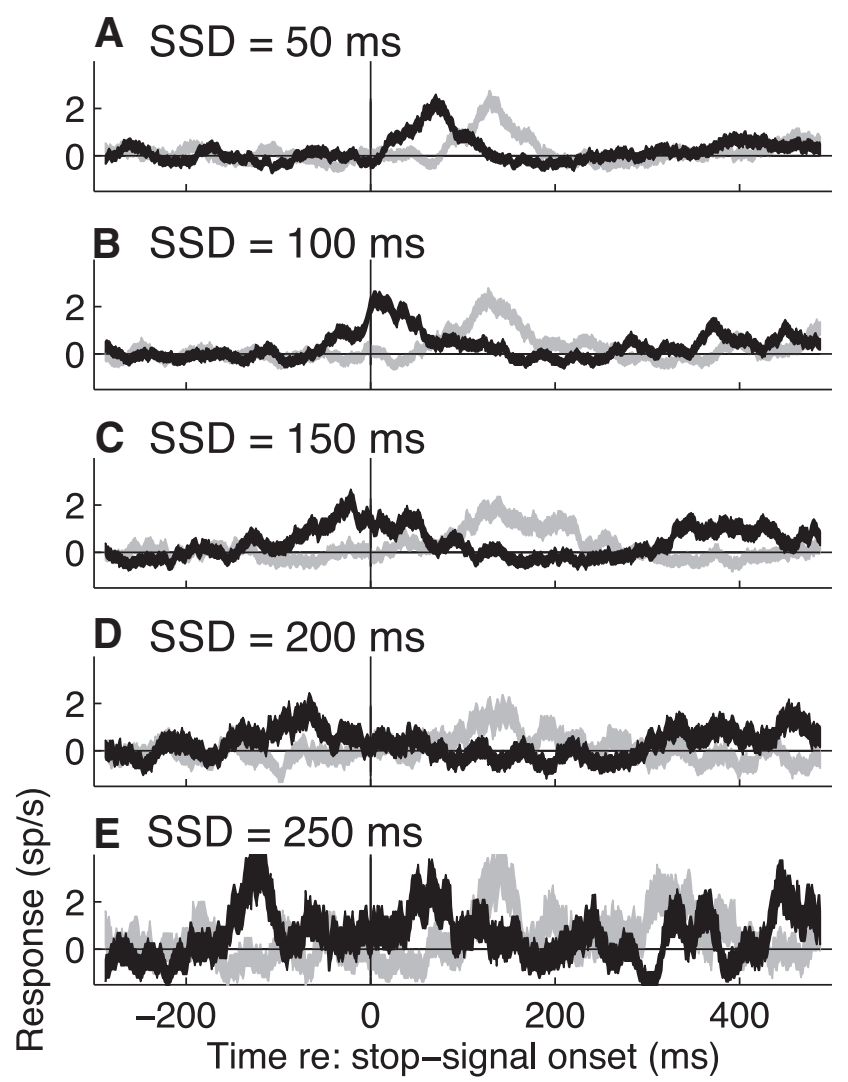

Figure 13. $L C+$ activation on correct-stop-signal trials. $\boldsymbol{A}-\boldsymbol{E}$ show the PETH for data combined from all single units for the indicated nominal stop-signal delay aligned to stop-signal onset. Vertical dashed lines indicate go-signal onset. For comparison, the PETH from the same units and the same trials, but aligned to go-signal onset (at time 0 ), is also shown (in gray).

driven decision not to act. Here, we discuss targeting $\mathrm{LC}+$ and then consider these findings in the context of previous studies of the timing of LC phasic responses and theories of LC function.

The small size of the LC and its location deep in the brainstem make it difficult to target for in vivo recordings, particularly in monkeys. Histological reconstruction and clonidine sensitivity can help but are typically used to confirm only a small subset of recording sites in a given experiment. Instead, most sites are targeted and identified using a combination of stereotaxic coordinates and a careful characterization of the response properties of neurons along each electrode penetration to distinguish LC from neighboring structures. Here we reported, and confirmed via histological reconstruction, that many of the response properties used to distinguish LC, including low baseline activity, broad spike waveforms, and arousal sensitivity, can also be consistent with the subC, an adjacent nucleus with NE-releasing neurons that project to the lower brainstem and spinal cord (Westlund and Coulter, 1980; Aston-Jones et al., 1994; Bouret and Sara, 2004; Bouret and Richmond, 2009). We do not know whether putative LC neurons recorded in other in vivo studies in monkeys also might have included subC units, nor were we able to distinguish LC and subC in our population of recorded units. We suspect that a careful functional mapping of units, as shown in Figure 3, along ML and AP axes of electrode penetrations might be used to distinguish a medial cluster as LC and a more lateral cluster as subC. Such AP and ML shifts can be assayed quantitatively using associated locations in the collicular saccade map along electrode penetrations (Robinson, 1972). A careful assessment of recording locations along the depth of each track may 
also prove to be informative because subC lies somewhat deeper than LC. However, we did not attempt this procedure for the present study and therefore group all of our units as LC + , leaving for future studies interesting questions about possible similarities and differences between LC and subC activation on sensorymotor tasks.

Early studies of LC response properties emphasized the efficacy of highly arousing sensory stimuli from different modalities in eliciting phasic LC responses (Foote et al., 1980; Aston-Jones and Bloom, 1981b; Grant et al., 1988; Hervé-Minvielle and Sara, 1995). These responses lead to NE release throughout the brain, including sensory areas, where it enhances the effects of incoming signals (Abercrombie et al., 1988; Servan-Schreiber et al., 1990; Waterhouse et al., 1998; Bouret and Sara, 2002; Devilbiss et al., 2006; Moxon et al., 2007; Devilbiss and Waterhouse, 2011). Other studies demonstrated that phasic LC activity depended on the goal-directed meaning of the stimuli, but upheld the view that these responses were correlated with the sensory events that led to subsequent behavior (Sara and Segal, 1991; Rajkowski et al., 2004). Therefore, the timing of LC phasic responses were typically interpreted in terms of their role in sensory processing, particularly as it related to attention and arousal (Berridge and Waterhouse, 2003).

More recent studies have suggested a closer relationship between LC phasic activation and motor output in simple sensorymotor tasks. In monkeys performing a go/no-go task that required them to pull a lever in response to an infrequently presented target stimulus and withhold the lever pull in response to a nontarget stimulus, LC neurons were consistently activated by target cues leading to a motor response. In contrast, the same LC neurons did not respond reliably to nontarget cues, juice rewards, or similar movements made outside of the task context. The results were interpreted in terms of a relationship between LC activation and "attended stimuli that demand a rapid motor response” (Aston-Jones et al., 1994). In monkeys performing a visual-discrimination task with variable RTs, LC spike timing was more closely aligned to the motor event than the sensory event, leading to speculation that LC responses represent the end of a decision process that leads to a behavioral response (Clayton et al., 2004). LC spikes were similarly timed relative to an operant motor response on a simple conditioning task, although phasic activation was also reported for the preceding, conditioned visual cue that was also accompanied by Pavlovian motor responses (Bouret and Richmond, 2009).

Despite these conflicting interpretations of LC activity in terms of sensory or motor processing, a common finding has been the presence of LC phasic responses only within the context of a behavior for which the animal is rewarded. For example, actions that activate LC neurons in the context of a task typically do not cause comparable activations outside of the task context (Yamamoto and Ozawa, 1989; Clayton et al., 2004). This context dependence is thought to involve reward expectation and/or uncertainty, which is also more consistent with the decision to act than the action itself (Sara and Segal, 1991; Aston-Jones et al., 1994; Vankov et al., 1995; Clayton et al., 2004; Rajkowski et al., 2004). Therefore, our finding that some LC units show phasic activation corresponding to saccades in the intertrial interval might still reflect task-related processing insofar as the monkeys were trained to expect a potentially rewarding trial to begin soon after the previous trial and thus might have been making an anticipatory movement to begin the next trial.

Our study clarifies and extends these previous findings by demonstrating that individual LC + units can be activated phasi- cally in relation to both a sensory "go" signal and the immediately resulting motor response. Our ability to distinguish these sensory- and motor-related activations, in contrast to previous studies, probably reflected our oculomotor task that produced shorter, more narrowly distributed RTs than lever-release tasks used previously (Clayton et al., 2004). We draw several conclusions from our findings, including: (1) sensory-evoked phasic responses in $\mathrm{LC}+$ do not require an overt motor output, because they were present on correct-stop-signal trials; (2) at least under our task conditions, motor-related activation typically does not occur until after movement onset and thus occurs too late to affect the current movement; and (3) the LC+ does not respond to a either a sensory instruction or the subsequent decision not to move even when a reward is expected, implying that reward expectation alone does not drive LC responses.

These results can be interpreted in the context of models of countermanding. One well established class of models assumes that there is a race between "go" and "stop" processes. Both processes are assumed to involve linear buildup signals that are triggered by the relevant sensory event and race toward a common threshold value (Logan and Cowan, 1984; Boucher et al., 2007; Verbruggen and Logan, 2009). The "go" rise-to-threshold process has clear correlates in the activity of particular sets of neurons in the primate frontal eye field and superior colliculus (Hanes and Schall, 1996; Hanes et al., 1998; Pare and Hanes, 2003). The sensory and motor phasic LC responses that we measured on no-stop-signal and incorrect-stop-signal trials correspond approximately to the beginning and end of this presaccadic buildup of neural activity. The neural implementation of the stop process is less clear, but might involve increased discharge of fixation neurons in the frontal eye field and superior colliculus (Hanes et al., 1998; Pare and Hanes, 2003; Boucher et al., 2007). Our results indicate a lack of LC + activation around the time of either onset or termination of this process. Therefore, the $\mathrm{LC}+$ is more engaged by the go than by the stop rise-to-threshold process posited by these models.

Alternatively, a recent model suggests that instead of a separate, rise-to-threshold stop process, withholding the planned saccade involves a rapid perceptual judgment that terminates the rise-to-threshold go process (Salinas and Stanford, 2013). This model would imply that the task-relevant sensory and motor phasic LC+ responses that we measured corresponded to the start and threshold crossing (i.e., commitment to trigger the saccadic eye movement) of the go process on no-stop-signal and incorrect-stop-signal trials. In contrast, the lack of responsiveness of LC+ neurons to stop-signal onset or the commitment to stop on correct-stop-signal trials might reflect the fact that, according to this model, there is no rise-to threshold stop process. More work is needed to establish more direct relationships between LC+ activation and the go and stop processes described by these models.

More generally, we do not know the function of the sensoryand motor-related phasic LC+ activation in the context of the countermanding task. The similarity of the sensory-related activation on all trial types, particularly when comparing correct and incorrect-stop-signal trials (Fig. 8), suggests that, at least on a gross level, this activation is not related to whether the monkeys subsequently process the stop signal appropriately. Moreover, the motor-related response occurs on both correct no-stopsignal trials and incorrect-stop-signal trials, is similar across different RTs, and typically occurs just after saccade onset. These results imply that the subsequent NE release does not affect the current motor response, but instead is intended to affect subse- 
quent processing, possibly in the context of evaluating the consequences of the goal-driven movement.

\section{References}

Abercrombie ED, Keller RW Jr, Zigmond MJ (1988) Characterization of hippocampal norepinephrine release as measured by microdialysis perfusion: pharmacological and behavioral studies. Neuroscience 27:897-904. CrossRef Medline

Aston-Jones G, Bloom FE (1981a) Activity of norepinephrine-containing locus coeruleus neurons in behaving rats anticipates fluctuations in the sleep-waking cycle. J Neurosci 1:876-886. Medline

Aston-Jones G, Bloom FE (1981b) Norepinephrine-containing locus coeruleus neurons in behaving rats exhibit pronounced responses to nonnoxious environmental stimuli. J Neurosci 1:887-900. Medline

Aston-Jones G, Cohen JD (2005) An integrative theory of locus coeruleusnorepinephrine function: adaptive gain and optimal performance. Annu Rev Neurosci 28:403-450. CrossRef Medline

Aston-Jones G, Rajkowski J, Kubiak P, Alexinsky T (1994) Locus coeruleus neurons in monkey are selectively activated by attended cues in a vigilance task. J Neurosci 14:4467-4480. Medline

Aston-Jones G, Rajkowski J, Kubiak P (1997) Conditioned responses of monkey locus coeruleus neurons anticipate acquisition of discriminative behavior in a vigilance task. Neuroscience 80:697-715. CrossRef Medline

Aston-Jones G, Rajkowski J, Cohen J (1999) Role of locus coeruleus in attention and behavioral flexibility. Biol Psychiatry 46:1309-1320. CrossRef Medline

Behrens F, Mackeben M, Schröder-Preikschat W (2010) An improved algorithm for automatic detection of saccades in eye movement data and for calculating saccade parameters. Behav Res Methods 42:701-708. CrossRef Medline

Berridge CW, Waterhouse BD (2003) The locus coeruleus-noradrenergic system: modulation of behavioral state and state-dependent cognitive processes. Brain Res Rev 42:33-84. CrossRef Medline

Boucher L, Palmeri TJ, Logan GD, Schall JD (2007) Inhibitory control in mind and brain: an interactive race model of countermanding saccades. Psychol Rev 114:376-397. CrossRef Medline

Bouret S, Richmond BJ (2009) Relation of locus coeruleus neurons in monkeys to Pavlovian and operant behaviors. J Neurophysiol 101:898-911. CrossRef Medline

Bouret S, Sara SJ (2002) Locus coeruleus activation modulates firing rate and temporal organization of odour-induced single-cell responses in rat piriform cortex. Eur J Neurosci 16:2371-2382. CrossRef Medline

Bouret S, Sara SJ (2004) Reward expectation, orientation of attention and locus coeruleus-medial frontal cortex interplay during learning. Eur J Neurosci 20:791-802. CrossRef Medline

Bouret S, Sara SJ (2005) Network reset: a simplified overarching theory of locus coeruleus noradrenaline function. Trends Neurosci 28:574-582. CrossRef Medline

Cahill L, McGaugh JL (1996) Modulation of memory storage. Curr Opin Neurobiol 6:237-242. CrossRef Medline

Carpenter RH, Williams ML (1995) Neural computation of log likelihood in control of saccadic eye movements. Nature 377:59-62. CrossRef Medline

Carter ME, Yizhar O, Chikahisa S, Nguyen H, Adamantidis A, Nishino S, Deisseroth K, de Lecea L (2010) Tuning arousal with optogenetic modulation of locus coeruleus neurons. Nat Neurosci 13:1526-1533. CrossRef Medline

Clayton EC, Rajkowski J, Cohen JD, Aston-Jones G (2004) Phasic activation of monkey locus ceruleus neurons by simple decisions in a forced-choice task. J Neurosci 24:9914-9920. CrossRef Medline

Corbetta M, Patel G, Shulman GL (2008) The reorienting system of the human brain: from environment to theory of mind. Neuron 58:306-324. CrossRef Medline

Cox RW (1996) AFNI: software for analysis and visualization of functional magnetic resonance neuroimages. Comput Biomed Res 29:162-173. CrossRef Medline

Devauges V, Sara SJ (1990) Activation of the noradrenergic system facilitates an attentional shift in the rat. Behav Brain Res 39:19-28. CrossRef Medline

Devilbiss DM, Waterhouse BD (2011) Phasic and tonic patterns of locus coeruleus output differentially modulate sensory network function in the awake rat. J Neurophysiol 105:69-87. CrossRef Medline
Devilbiss DM, Page ME, Waterhouse BD (2006) Locus ceruleus regulates sensory encoding by neurons and networks in waking animals. J Neurosci 26:9860-9872. CrossRef Medline

Emeric EE, Brown JW, Boucher L, Carpenter RH, Hanes DP, Harris R, Logan GD, Mashru RN, Paré M, Pouget P, Stuphorn V, Taylor TL, Schall JD (2007) Influence of history on saccade countermanding performance in humans and macaque monkeys. Vision Res 47:35-49. CrossRef Medline

Foote SL, Aston-Jones G, Bloom FE (1980) Impulse activity of locus coeruleus neurons in awake rats and monkeys is a function of sensory stimulation and arousal. Proc Natl Acad Sci U S A 77:3033-3037. CrossRef Medline

Grant SJ, Aston-Jones G, Redmond DE Jr (1988) Responses of primate locus coeruleus neurons to simple and complex sensory stimuli. Brain Res Bull 21:401-410. CrossRef Medline

Hanes DP, Carpenter RH (1999) Countermanding saccades in humans. Vision Res 39:2777-2791. CrossRef Medline

Hanes DP, Schall JD (1995) Countermanding saccades in macaque. Vis Neurosci 12:929-937. CrossRef Medline

Hanes DP, Schall JD (1996) Neural control of voluntary movement initiation. Science 274:427-430. CrossRef Medline

Hanes DP, Patterson WF 2nd, Schall JD (1998) Role of frontal eye fields in countermanding saccades: visual, movement, and fixation activity. J Neurophysiol 79:817-834. Medline

Harley CW (1987) A role for norepinephrine in arousal, emotion and learning?: limbic modulation by norepinephrine and the Kety hypothesis. Prog Neuropsychopharmacol Biol Psychiatry 11:419-458. CrossRef Medline

Henn V, Büttner-Ennever JA, Hepp K (1982) The primate oculomotor system. I. Motoneurons. A synthesis of anatomical, physiological, and clinical data. Hum Neurobiol 1:77-85. Medline

Hervé-Minvielle A, Sara SJ (1995) Rapid habituation of auditory responses of locus coeruleus cells in anaesthetized and awake rats. Neuroreport 6:1363-1368. CrossRef Medline

Hill DN, Mehta SB, Kleinfeld D (2011) Quality metrics to accompany spike sorting of extracellular signals. J Neurosci 31:8699-8705. CrossRef Medline

Howells FM, Stein DJ, Russell VA (2012) Synergistic tonic and phasic activity of the locus coeruleus norepinephrine (LC-NE) arousal system is required for optimal attentional performance. Metab Brain Dis 27:267-274. CrossRef Medline

Jones BE, Moore RY (1977) Ascending projections of the locus coeruleus in the rat. II. Autoradiographic study. Brain Res 127:25-53. Medline

Jouvet M (1969) Biogenic amines and the states of sleep. Science 163:32-41. CrossRef Medline

Kalwani RM, Bloy L, Elliott MA, Gold JI (2009) A method for localizing microelectrode trajectories in the macaque brain using MRI. J Neurosci Methods 176:104-111. CrossRef Medline

Logan GD, Cowan WB (1984) On the ability to inhibit thought and action: a theory of an act of control. Psychol Rev 91:295-327. CrossRef

Logan GD, Cowan WB, Davis KA (1984) On the ability to inhibit simple and choice reaction time responses: a model and a method. J Exp Psychol Hum Percept Perform 10:276-291. CrossRef Medline

Moxon KA, Devilbiss DM, Chapin JK, Waterhouse BD (2007) Influence of norepinephrine on somatosensory neuronal responses in the rat thalamus: a combined modeling and in vivo multi-channel, multi-neuron recording study. Brain Res 1147:105-123. CrossRef Medline

Nelson MJ, Boucher L, Logan GD, Palmeri TJ, Schall JD (2010) Nonindependent and nonstationary response times in stopping and stepping saccade tasks. Atten Percept Psychophys 72:1913-1929. CrossRef Medline

Paré M, Hanes DP (2003) Controlled movement processing: superior colliculus activity associated with countermanded saccades. J Neurosci 23 : 6480-6489. Medline

Paxinos G, Huang X-F, Petrides M, Toga A (2008) The Rhesus monkey brain: in stereotaxic coordinates.

Pouget P, Logan GD, Palmeri TJ, Boucher L, Paré M, Schall JD (2011) Neural basis of adaptive response time adjustment during saccade countermanding. J Neurosci 31:12604-12612. CrossRef Medline

Rajkowski J, Majczynski H, Clayton E, Aston-Jones G (2004a) Activation of monkey locus coeruleus neurons varies with difficulty and performance in a target detection task. J Neurophysiol 92:361-371. CrossRef Medline

Rasmussen K, Morilak DA, Jacobs BL (1986) Single unit activity of locus coeruleus neurons in the freely moving cat. I. During naturalistic behav- 
iors and in response to simple and complex stimuli. Brain Res 371:324334. CrossRef Medline

Rieger M, Gauggel S (1999) Inhibitory after-effects in the stop signal paradigm. Br J Psychol 90:509-518. CrossRef

Robinson DA (1972) Eye movements evoked by collicular stimulation in the alert monkey. Vision Res 12:1795-1808. CrossRef Medline

Salinas E, Stanford TR (2013) The countermanding task revisited: fast stimulus detection is a key determinant of psychophysical performance. J Neurosci 33:5668-5685. CrossRef Medline

Sara SJ (2009) The locus coeruleus and noradrenergic modulation of cognition. Nat Rev Neurosci 10:211-223. CrossRef Medline

Sara SJ, Bouret S (2012) Orienting and reorienting: the locus coeruleus mediates cognition through arousal. Neuron 76:130-141. CrossRef Medline

Sara SJ, Segal M (1991) Plasticity of sensory responses of locus coeruleus neurons in the behaving rat: implications for cognition. Prog Brain Res 88:571-585. CrossRef Medline

Segal M, Foote SL, Aston-Jones G (1983) Physiological properties of ascending locus coeruleus axons in the squirrel monkey. Brain Res 274:381387. CrossRef Medline

Servan-Schreiber D, Printz H, Cohen JD (1990) A network model of catecholamine effects: gain, signal-to-noise ratio, and behavior. Science 249: 892-895. CrossRef Medline

Sharma Y, Xu T, Graf WM, Fobbs A, Sherwood CC, Hof PR, Allman JM, Manaye KF (2010) Comparative anatomy of the locus coeruleus in humans and nonhuman primates. J Comp Neurol 518:963-971. CrossRef Medline

Smeets WJ, González A (2000) Catecholamine systems in the brain of ver- tebrates: new perspectives through a comparative approach. Brain Res Brain Res Rev 33:308-379. CrossRef Medline

Sparks DL, Nelson JS (1987) Sensory and motor maps in the mammalian superior colliculus. Trends Neurosci 10:312-317. CrossRef

Usher M, Cohen JD, Servan-Schreiber D, Rajkowski J, Aston-Jones G (1999) The role of locus coeruleus in the regulation of cognitive performance. Science 283:549-554. CrossRef Medline

Vankov A, Hervé-Minvielle A, Sara SJ (1995) Response to novelty and its rapid habituation in locus coeruleus neurons of the freely exploring rat. Eur J Neurosci 7:1180-1187. CrossRef Medline

Verbruggen F, Logan GD (2008) Response inhibition in the stop-signal paradigm. Trends Cogn Sci.

Verbruggen F, Logan GD (2009) Models of response inhibition in the stopsignal and stop-change paradigms. Neurosci Biobehav Rev 33:647-661. CrossRef Medline

Waterhouse BD, Moises HC, Woodward DJ (1998) Phasic activation of the locus coeruleus enhances responses of primary sensory cortical neurons to peripheral receptive field stimulation. Brain Res 790:33-44. CrossRef Medline

Westlund KN, Coulter JD (1980) Descending projections of the locus coeruleus and subcoeruleus/medial parabrachial nuclei in monkey: axonal transport studies and dopamine-beta-hydroxylase immunocytochemistry. Brain Res 2:235-264. CrossRef Medline

Yamamoto K, Ozawa N (1989) Increased firing of locus coeruleus neurons associated with preparatory set in rats. Neurosci Lett 106:112-118. CrossRef Medline 